\title{
New microstructural insights into neutron-irradiated tungsten
}

\author{
Michael Dürrschnabel \\ Karlsruhe Institute of Technology \\ Michael Klimenkov ( $\nabla$ michael.klimenkov@kit.edu ) \\ Karlsruhe Institute of Technology \\ Ute Jäntsch \\ Karlsruhe Institute of Technology \\ Michael Rieth \\ Karlsruhe Institute of Technology \\ Hans-Christian Schneider \\ Karlsruhe Institute of Technology \\ Dmitry Terentyev \\ Belgian Nuclear Research Centre
}

\section{Research Article}

Keywords: tungsten, neutron irradiation, microstructure, transmutation, voids, chi phase, sigma phase, irradiation defects

Posted Date: December 10th, 2020

DOl: https://doi.org/10.21203/rs.3.rs-119608/v1

License: (c) (i) This work is licensed under a Creative Commons Attribution 4.0 International License. Read Full License

Version of Record: A version of this preprint was published at Scientific Reports on April 7th, 2021. See the published version at https://doi.org/10.1038/s41598-021-86746-6. 


\title{
New microstructural insights into neutron-irradiated tungsten
}

\author{
M. Dürrschnabel ${ }^{1}$, M. Klimenkov ${ }^{1, *}$, U. Jäntsch ${ }^{1}$, M. Rieth ${ }^{1}$, H.C. Schneider ${ }^{2}$ D. Terentyev ${ }^{3}$ \\ ${ }^{1}$ Karlsruhe Institute of Technology (KIT), Institute for Applied Materials- Applied Materials \\ Physics, 76021 Karlsruhe, Germany \\ ${ }^{2}$ Karlsruhe Institute of Technology (KIT), Institute for Applied Materials - Materials- and \\ Biomechanics, 76021 Karlsruhe, Germany \\ ${ }^{3}$ SCK CEN, Nuclear Materials Science Institute, Boeretang 200, 2400 Mol, Belgium \\ * corresponding author, contact: michael.klimenkov@kit.edu
}

\section{Abstract}

The development of appropriate materials for fusion reactors that can sustain high neutron fluencies at elevated temperatures remains a great challenge. Tungsten is one of the promising candidate materials for plasma-facing components of future fusion reactors, due to several favorable properties as for example a high melting point, a high sputtering resistivity, and a low coefficient of thermal expansion. The microstructural details of a tungsten sample with a 1.25 dpa (displacements per atom) damage dose after irradiation at $800^{\circ} \mathrm{C}$ were examined by transmission electron microscopy (TEM). Three types of radiation-induced defects were observed, analyzed and characterized: (i) voids with sizes ranging from $10 \mathrm{~nm}$ to $65 \mathrm{~nm}$, (ii) dislocation loops with a size of up to $10 \mathrm{~nm}$ and (iii) $\mathrm{W}$-Re-Os containing $\sigma$ - and $\chi$-type precipitates. The distribution of voids as well as the nature of the occurring dislocation loops were studied in detail. In addition, nano-chemical analyses revealed that the $\sigma$ - and $\chi$-type precipitates, which are sometimes attached to voids, are surrounded by a solid solution cloud enriched with Re. For the first time the crystallographic orientation relationship of the $\sigma$ - and $\chi$-phases to the $\mathrm{W}$-matrix was specified. Furthermore, electron energy-loss spectroscopy could not unambiguously verify the presence of He within individual cavities.

Key words: tungsten, neutron irradiation, microstructure, transmutation, voids, chi phase, sigma phase, irradiation defects 


\section{Introduction}

Tungsten (W) is the most cited material candidate for high-temperature vacuum or inert gas applications in power technology. Due to its favorable properties, such as the highest melting point in combination with high creep resistance, very high sputter resistance, a low coefficient of thermal expansion and a rather high thermal conductivity, it theoretically allows the raising of process temperatures into previously unknown temperature ranges. Besides renewable power generation methods, such as for example concentrated solar power, future fusion reactors will greatly benefit in particular by the use of $\mathrm{W}$ in plasma-facing structural parts. In the latter case, the material has to withstand very high operation temperatures as well as it has to tolerate a significant amount of neutron-induced radiation damage ${ }^{1-3}$. A detailed experimental understanding of the neutron damage processes on the nanoscale, which is still limited at present time, is of utmost importance in the material qualification process.

Extensive microstructural characterizations of $\mathrm{W}$ have already been carried out to understand the microstructural response to neutron irradiation ${ }^{3-8}$. However, the covered irradiation doses and temperature range in these investigations are limited. Increasing both parameters to the conditions expected in a future fusion reactor will address still unsolved problems regarding the microstructure.

Basically, there are three mechanisms that may degrade the beneficial material properties of $\mathrm{W}$ during neutron irradiation: (1) Firstly, this includes the formation of lattice defects such as Frenkel pairs, interstitial and vacancy clusters and dislocation loops. (2) In addition, the formation of voids leads to swelling and radiation hardening that would restrict the lifetime of components beyond certain design limits. (3) The transmutation processes also lead to the formation of rhenium- ( $\mathrm{Re}$ ) or osmium- (Os) rich phases, while helium transmutation could stabilize voids. Both effects can lead to further significant embrittlement of $\mathrm{W}$ materials.

An additional issue arises with the experimental determination of the occurrence and dynamics of irradiation defects in material (fission) test reactors. They do not only depend on the neutron dose and temperature, but also on the transmutation rates, which themselves strongly hinge on the reactor type and the exact capsule position within the active reactor zone. As a consequence, each irradiation experiment has to be considered as a unique result, which paves the way for a more complete systematization and prediction of neutron radiation damage.

In contrast to technologically relevant neutron doses, irradiation in the sub-dpa range does not lead to a significant accumulation of Re or Os. In former studies, W-Re and W-Re-Os alloys were neutron-irradiated in order to simulate the influence of transmutation-induced elements on the microstructure and mechanical properties ${ }^{9-11}$. As was shown, the presence of $\mathrm{Re}$ influences the formation of voids and, thus, may contribute further to the hardening of the material. However, the obvious different behavior of Re and Os as solid solution in W opposed to their occurrence in form of transmuted elements is not yet fully understood. Therefore, in this work we focus not only on the comprehensive characterization of radiation induced loops and voids but also on the precipitation of Re- and Os-containing $\sigma-$ and $\chi$-phases. Moreover, as far as possible we examine and analyze the influence of grain boundaries on formation of these defects. Finally, to maximize the output of the investigation on a set of unique irradiation samples, we make use of the recent progress in nanoanalytical methods implemented in transmission electron microscopy (TEM) such as for example the ChemiSTEM ${ }^{\mathrm{TM}}$ technology, 
high-speed DualEELS, and high-performance CCD cameras in order to understand the above mentioned neutron irradiation effects on technological $\mathrm{W}$ grade in more detail.

\section{Experimental}

The polycrystalline $\mathrm{W}$ was neutron irradiated up to a damage dose of $1.25 \mathrm{dpa}$ at $800^{\circ} \mathrm{C}$ in the BR2 reactor located in Mol (Belgium). Stainless steel thick-walled capsules were implemented to reduce the flux of thermal neutrons, and the irradiation device has been placed inside the fuel element to maximize the fast-to-thermal neutron ratio. The total irradiation time was 143 days and the neutron flux was $510^{14}(\mathrm{E}>0.1 \mathrm{MeV}), 210^{14}(\mathrm{E}>1 \mathrm{MeV})$ and $4.210^{14}(\mathrm{E}<0.1 \mathrm{MeV})$ $\mathrm{n} / \mathrm{cm}^{2} / \mathrm{s}$. The transmutation induced content of Re and Os was determined to be $\sim 2$ at. $\%$ and 0.2 at. $\%$, respectively.

Since the material is radioactive, it is advantageous to use a focused ion beam machine (FIB, FEI Scios ) for specimen preparation to (i) limit the radiation exposure and (ii) the specimen preparation in a predefined area. Thin foils for TEM investigations were prepared using the FIB technique in Fusion Materials Laboratory (FML) at KIT. The thin lamellae were attached to a molybdenum grid. After preparation the lamella was flash polished in $1 \% \mathrm{NaOH}$ water solution using method described in the ref. ${ }^{12}$.

TEM analysis was performed in a Thermofisher Talos F200X scanning transmission electron microscope (STEM) equipped with four energy-dispersive X-ray (EDX) detectors. STEMEDX maps were acquired in the Velox software using $512 \times 512$ pixels and a spectral dispersion of $5 \mathrm{eV}$. The EDX detector resolution is specified by the manufacturer as $\leq 136 \mathrm{eV}$ at $\mathrm{Mn}-\mathrm{K}_{\alpha}$. At the $\mathrm{W}-\mathrm{L}_{\alpha}(\mathrm{E}=8.396 \mathrm{keV})$ the energy resolution has a value of about $150-160 \mathrm{eV}$, which is sufficient to separate the W- $\mathrm{L}_{\alpha}$, Re- $\mathrm{L}_{\alpha}$ and Os- $\mathrm{L}_{\alpha}$ X-ray lines. Quantification of STEM-EDX data was done using the Cliff-Lorimer k-factor method. The TEM images and selected area diffraction pattern (SAED) were acquired by using a Thermofisher Ceta 16M CCD camera. The STEM-EELS data was acquired using a convergence angle of $10.5 \mathrm{mrad}$ and a collection angle of $14.1 \mathrm{mrad}$. Furthermore, the extraction voltage was reduced such that the final energy resolution was about $0.7 \mathrm{eV}$.

Table 1: Summary of crystal structures used for JEMS modelling. The * indicates that the ICSD number belongs to the WRe3 structure.

\begin{tabular}{llll}
\hline Phase & ICSD No. & Space group & Lattice \\
\hline $\mathbf{W}$ & 43421 & $\mathrm{Im}-3 \mathrm{~m}$ & cubic, $\mathrm{bcc}, \mathrm{a}=3.17 \AA$ \\
$\boldsymbol{\sigma}$-WOs 2 & 150547 & $\mathrm{P} 42 / \mathrm{mnm}$ & $\begin{array}{l}\text { tetragonal } \\
\mathrm{a}=\mathrm{b}=9.43 \AA \mathrm{c}=4.99 \AA\end{array}$ \\
$\boldsymbol{\chi}$-WOs $_{3}$ & $650196^{*}$ & $\mathrm{I}-43 \mathrm{~m}$ & cubic, $\mathrm{a}=9.60 \AA$ \\
\hline
\end{tabular}

The diffractograms obtained from high-resolution phase contrast images were fitted using JEMS software package ${ }^{13}$. The crystallographic structures of the individual phases that were used as input are summarized in Table 1 . In case of the WOs3 $\chi$-phase no structural model was 
available, therefore, we used $\mathrm{WRe}_{3} \chi$-phase and replaced Re by Os, which should be feasible since atomic radii of both atoms differ only by a few picometers ${ }^{14}$.

Data post processing was performed using the HyperSpy software package ${ }^{15}$ and Gatan Digital Micrograph. Independent component analysis (ICA) was performed via the FastICA algorithm on the results of a principal component analysis (PCA) as implemented in the Hyperspy package. The data was normalized during PCA with respect to Poisson statistics. The STEMEDX data was binned by a factor of two in spectral direction prior to PCA analysis.

The analysis of the Burgers vector of dislocation loops includes dark field imaging under defined orientation of the g-vector near the [110] zone axis. The visibility criteria for dislocation loops with $\mathrm{b}^{1 / 2}<111>$ and $<100>$ are given in the Table 2 .

\begin{tabular}{lccccccc}
\hline$g \backslash b$ & $\mathbf{1} / \mathbf{2}[\mathbf{1 1 1}]$ & $\mathbf{1} / \mathbf{2}[\overline{\mathbf{1}} \mathbf{1 1}]$ & $\mathbf{1} \mathbf{2}[\mathbf{1 \mathbf { 1 }} 1]$ & $\mathbf{1} / \mathbf{2}[\mathbf{1 1} \overline{\mathbf{1}}]$ & {$[\mathbf{0 0 1}]$} & {$[\mathbf{0 1 0}]$} & {$[\mathbf{1 0 0}]$} \\
\hline$[\overline{\mathbf{1 1}} \mathbf{1 0}]$ & 0 & 1 & 1 & 0 & 0 & 1 & 1 \\
{$[\mathbf{0 0 2}]$} & 1 & 1 & 1 & 1 & 1 & 0 & 0 \\
\hline
\end{tabular}

Table 2: Visibility criteria for dislocation loops in diffraction contrast microscopy. The loops is visible if the absolute value of the scalar product $|\mathrm{b} \cdot \mathrm{g}|=1$, if it is 0 the loop is invisible in the image.

\section{Results}

The microstructure of "as-delivered" material was detailed characterized and published in refs. ${ }^{16,17}$. The investigated tungsten shows an average grain structure with a size of $88 \mu \mathrm{m}$. Detailed TEM analyses revealed the occurrence of three types of irradiation defects: (i) voids with a size ranging from $10 \mathrm{~nm}$ to $65 \mathrm{~nm}$, (ii) "black dots" and dislocation loops with a size of up to $10 \mathrm{~nm}$ and (iii) W-Re-Os containing $\sigma$ - and $\chi$-type precipitates. A detailed TEM characterization of all three defect types is presented in the next subsections. From the previous studies of neutron irradiated $\mathrm{W}$ and beryllium it is well known ${ }^{6,18}$ that the accumulation of irradiation defects near grain boundary and in the grain interior differs, i.e. so-called denuded zone is formed close to the grain boundary, which is depleted by or even completely free of any void. Therefore, it is important to examine the formation of radiation-induced defects in the two areas, i.e. grain interior and grain boundary.

\subsection{Distribution of voids}

Figure 1 shows TEM bright-field images of a representative grain interior, Figure 1a and a depicts a representative grain boundary (GB) region (Figure $1 \mathrm{~b}$ ). The voids that were generated by neutron irradiation appear in bright contrast. This was exploited to determine their size distribution as well as their number density. The local sample thickness needed to determine the number density was estimated via quantification of EDX spectra. In the grain interior (Figure 1a) voids with a maximum size of $65 \mathrm{~nm}$ and a number density of $(4.3 \pm 1.2) 10^{20} \mathrm{~m}^{-3}$ were found. In addition, the voids located in the grain interior are more or less homogeneously 
distributed. The inset in Figure 1a proves that the voids are also faceted, i.e. the facets are of (110)-type. As can be seen in Figure 1a, some voids are attached to W-Re-Os-rich precipitates having a darker contrast than the surrounding $\mathrm{W}$ matrix. A detailed analysis of these Re-Osrich precipitates is carried out in subsection 3.3.
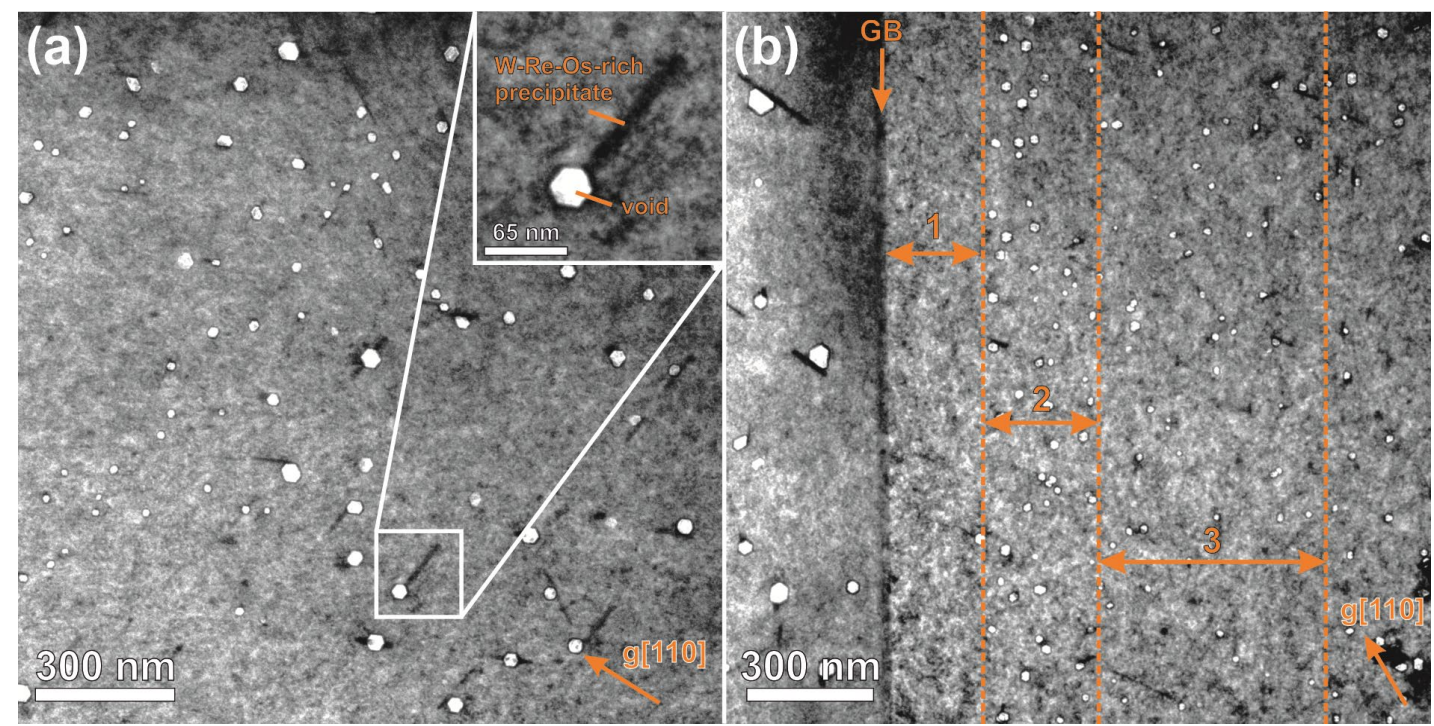

Figure 1: Bright-field TEM images acquired from neutron-irradiated W. (a) A representative area of a grain interior with voids (bright) and Re-Os-rich precipitates (dark strips). The inset shows a magnified view of a individual void attached to a W-Re-Os-rich precipitate. (b) A representative grain boundary (GB) region. The numbered areas are (1) the void denuded zone, (2) void-peak zone and (3) the second denuded zone.

At the grain boundary the spatial distribution of voids is markedly different than in the grain interior as can be seen by comparing features on Figure 1a and Figure 1b. Three different well distinguishable zones are found: the (1) void denuded zone, (2) void-peak zone and (3) the second denuded zone. The void denuded zone is directly attached to the grain boundary in the present case, it has a width of about $200 \mathrm{~nm}$ being completely free of voids (see Table 3 ). The "void peak zone" has a width of about $300 \mathrm{~nm}$. The notion "void peak zone" is due to the increased number density of voids, which is about twice as high as that determined in the grain interior as can be seen in Table 3. This zone is followed by the second denuded zone, which is about $500 \mathrm{~nm}$ in width. Here the void number density considerably decreases to about half of the value measured in the grain interior (see Table 3). The homogeneous distribution of voids, which is typical for the grain interior, is observed at $1 \mu \mathrm{m}$ away from grain boundary.

The size distribution histograms for voids registered in the grain interior (blue bars) and in the void peak zone (red bars) are shown in Figure 2. In the grain interior the voids have a lognormal-type size distribution as can be seen in Figure 2a, whereas in the void peak zone voids have Gaussian-like distribution profile. In the grain interior the void sizes are ranging from 8 $\mathrm{nm}$ to $60 \mathrm{~nm}$ and have an average size of $31 \mathrm{~nm}$. In the void peak zone, the void sizes are ranging from $14 \mathrm{~nm}$ to $39 \mathrm{~nm}$ with an average size of $24 \mathrm{~nm}$. 

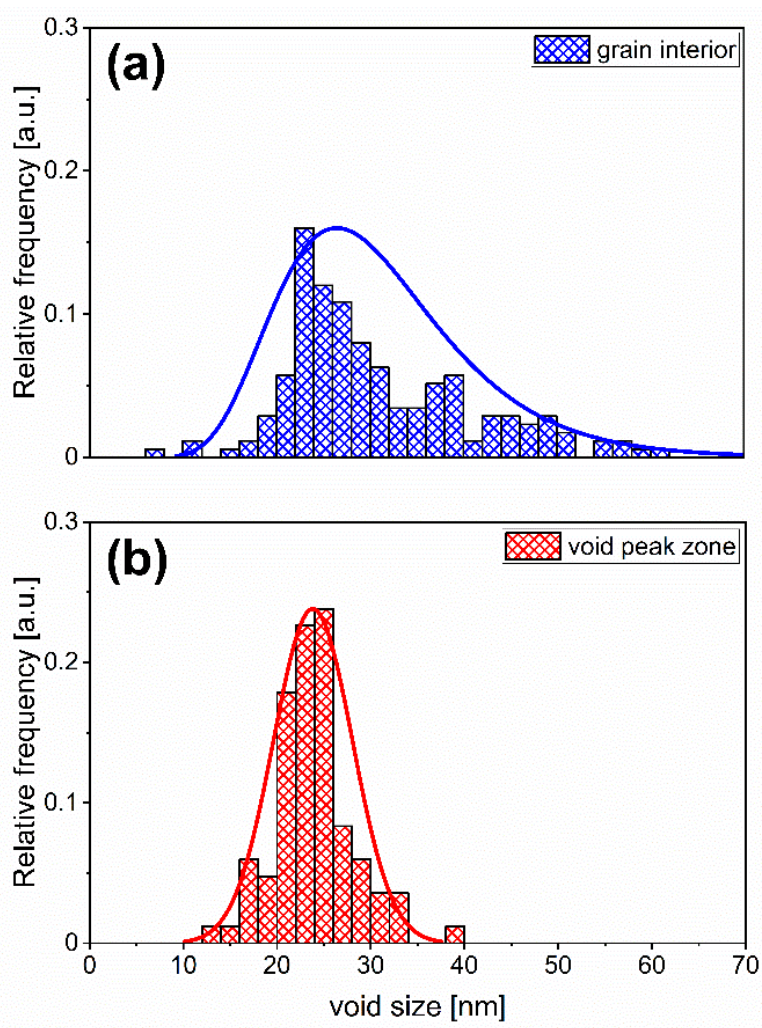

Figure 2: Size distribution histograms of the voids registered in the (a) grain interior and (b) void peak zone.

\subsection{Dislocation loops}
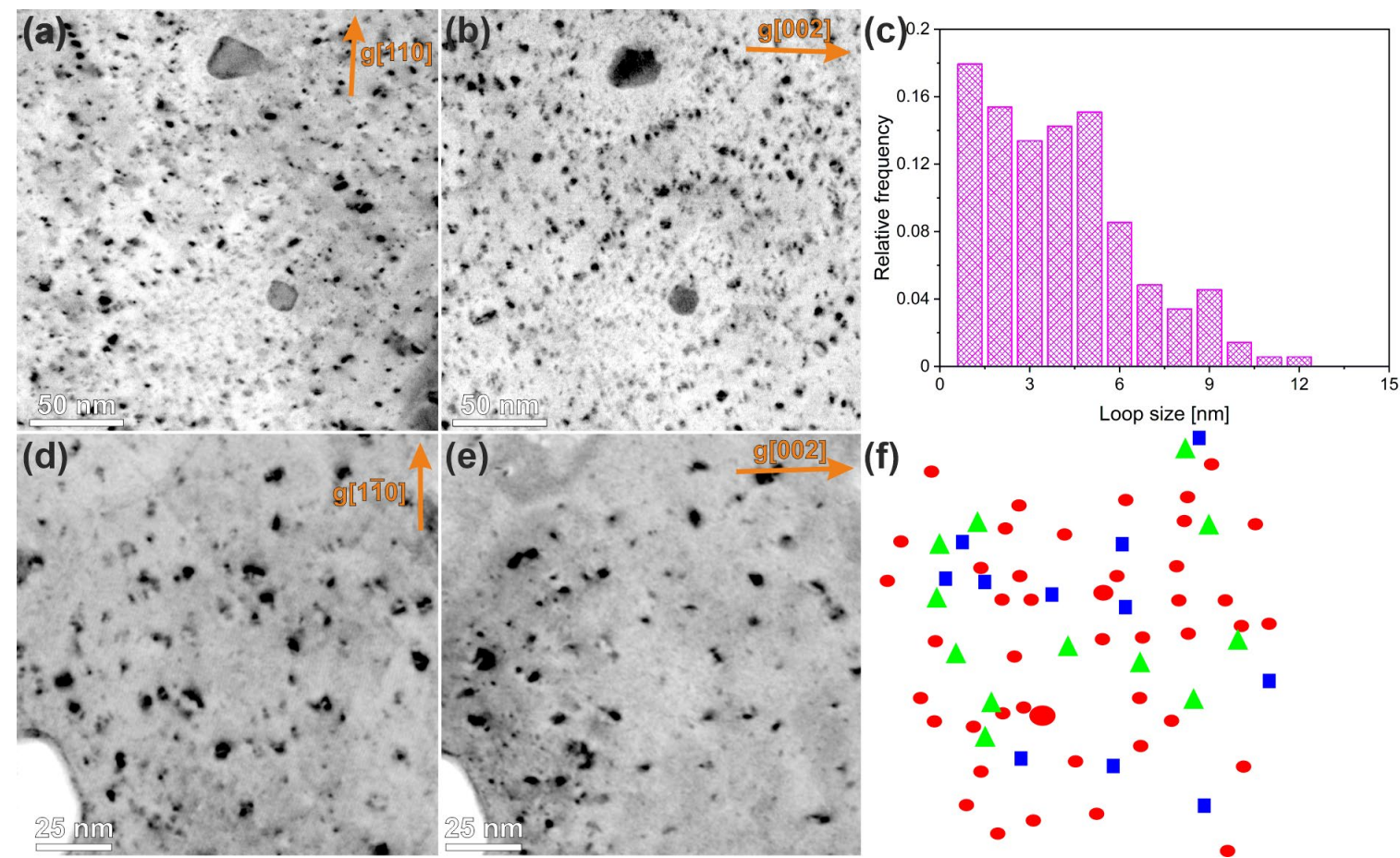

Figure 3: Visualization of dislocation loops and black dots in neutron-irradiated W resolved by reverse contrast dark-field (DF) imaging. DF images obtained with $\mathrm{g}[110]$ and $\mathrm{g}[002] \mathrm{g}$-vectors 
near the [110] zone-axis (a) and (b). These images were the basis for the loop size diagram (c). DF images obtained with g[110] and g[002] g-vectors near the [110] zone-axis (d) and (e). The loops with $b^{1} / 2\langle 111\rangle$ and $\mathrm{b}\langle 100\rangle$ Burgers vectors are imaged in the part (f) with red circles and blue squares, respectively. The loops with undefined Burgers vector are denoted by green triangles.

In addition to the voids, dislocation loops are also generated in considerable amount during neutron irradiation. These are observed mainly as small featureless black dots and circular loops. Figure 3 visualizes typical neutron-irradiation defects by reverse contrast dark-field (DF) imaging with defined g-vectors. The contrast in such images is uniform and less sensitive to local variations of sample thickness. Figures $3 a$ and $b$ show a general view of dislocation loops, which were imaged with different g-vectors near the [110] zone-axis. These images are the basis for the loop size histogram shown in Figure 3c. A considerable fraction of the defects are featureless black dots with a size of less than $6 \mathrm{~nm}$. The average size of all loops was measured to be $4.5 \mathrm{~nm}$. The calculation of the defect number density normalized to the local layer thickness leads to a value of about $(4.0 \pm 1.5) 10^{23} \mathrm{~m}^{-3}$. The uncertainty in the determination of local sample thickness is the reason for the $30 \%$ error of this value. The absence of dislocation loops in the 10-20 nm region around the precipitates can be observed. This indicates that the precipitates serve as sinks for interstitial atoms which inhibits the formation of the loops nearby 4 .

The high number density as well as the small size of the defects hamper the determination of the Burger's vectors in Figure $3 \mathrm{a}$ and $\mathrm{b}$ applying the visibility criteria or identification habit plane as presented in ${ }^{19}$. The value given in Table 3 was taken as the average of the two methods. The $N_{\langle 100\rangle} / N_{1 / 2\langle 111\rangle}$ ratio was obtained by the method described in ref. ${ }^{20}$ using the visible number densities for $\mathrm{g}[-110]$ and $\mathrm{g}[002]$ vectors. The result shows that $(25 \pm 20) \%$ of the defects have a $b\langle 100\rangle$ and $(75 \pm 20) \%$ a $b \frac{1}{2}\langle 111\rangle$ Burgers vector. To minimize the above-mentioned problems and to improve the statistics for the loops with a size of less than $3 \mathrm{~nm}$, the largemagnification reverse contrast DF images were acquired from a $40 \mathrm{~nm}$ thick area near the [110] zone axis (Figure $3 \mathrm{~d}$ and e). The Burgers vector of the defects was analyzed according to the visibility criteria and the possible habit plane (Table 2). The results of this analysis are shown with geometric shapes of different colors in Figure 3f. These methods show that $15 \%$ of the defects have a $b\langle 100\rangle$ and $85 \%$ a $b^{1 / 2}\langle 111\rangle$ Burger's vector. This finding is in good agreement with the results found on the basis of the number density. The value given in Table 3 was taken as the average of the two methods. 

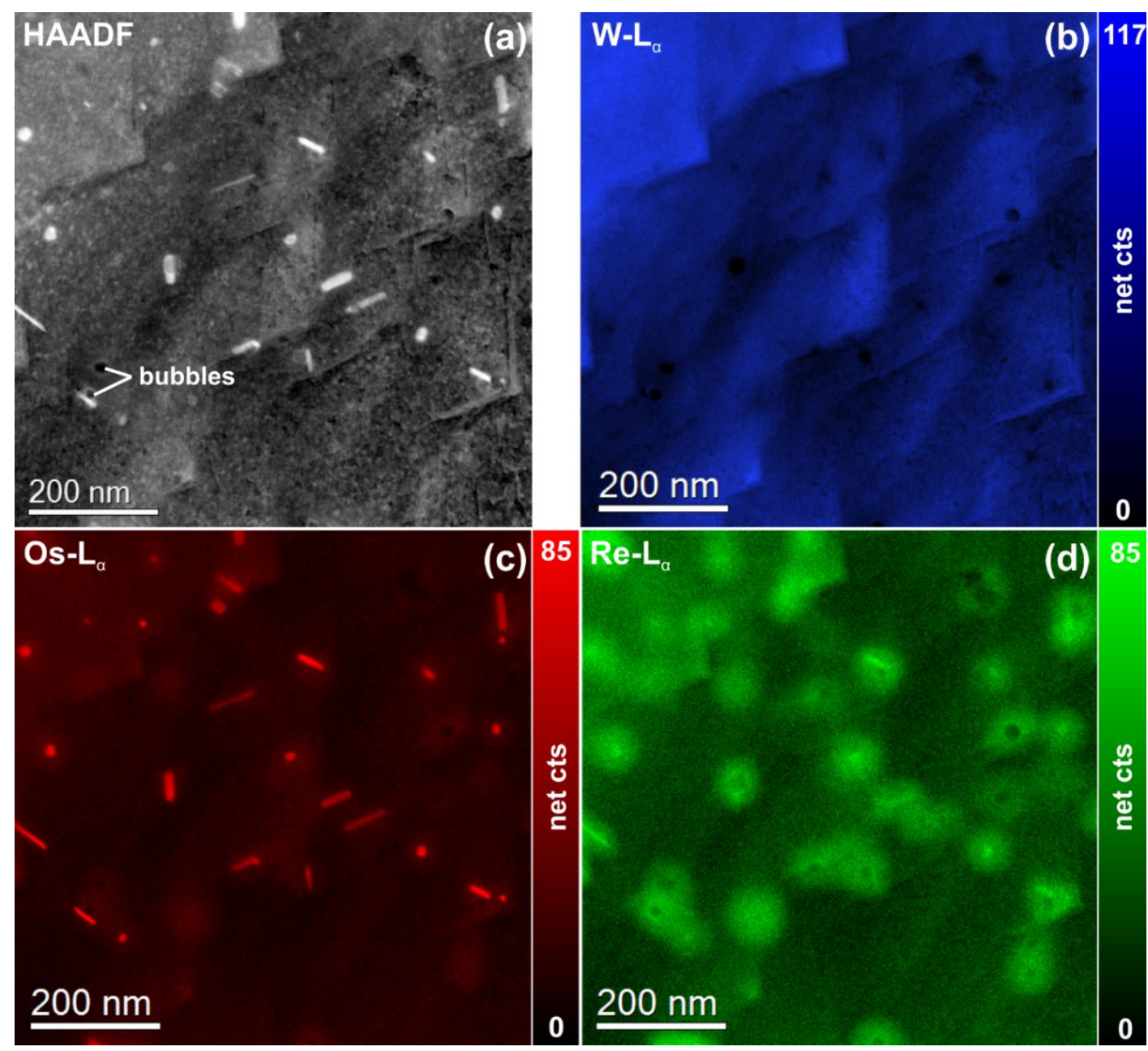

Figure 4: STEM-EDX spectrum image of a representative region in a neutron-irradiated W specimen. (a) STEM-HAADF image. Elemental maps of (b) W, (c) Os, and (d) Re display the location of elements with respect to the sample morphology. The tile-like structure in the HAADF image and the W map is surface topography due to flash polishing.

Figure 4 shows a STEM-EDX spectrum image of a representative region in a neutron-irradiated $\mathrm{W}$ specimen. The STEM-HAADF image in Figure 4 reveals that the precipitates are composed of atoms with $\mathrm{Z}$ number larger than the $\mathrm{W}$ matrix. It also shows that the sample has a surface topography due to selective etching during flash polishing, which is confirmed by the W-L map, see Figure $4 \mathrm{~b}$. The bright precipitates in the HAADF image are determined to be Os-rich particles as can be seen in the Os-L map, see Figure 4c. In addition, Figure 4c points that two types of precipitates co-exist: (i) rod-shaped ones with a size is $50-70 \mathrm{~nm}$ and (ii) spherical or polyhedral-shaped ones with a size of 15-20 nm. Furthermore, it is found that a Re-rich "cloudlike" phase is present around the Os-rich precipitates (see Re map in Figure 4d). The STEMHAADF image in Figure 4a exhibits no contrast change which indicates that the Re-enriched region has a coherent to matrix structure. The size of these Re-rich "clouds" depends on the size of the Os-rich core as well as on the density of the precipitates (i.e. in case of closely spaced Os-rich particles the respective Re-clouds overlap) and typically it is about $100 \mathrm{~nm}$. 
Furthermore, some voids with attached to the Os-rich precipitates were observed within the W matrix.

(a)

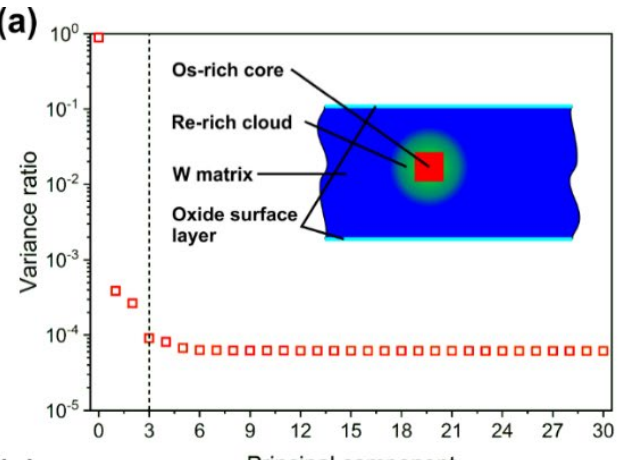

(c)

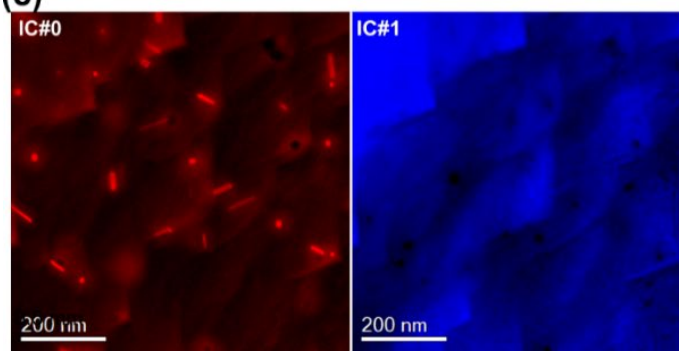

(b)

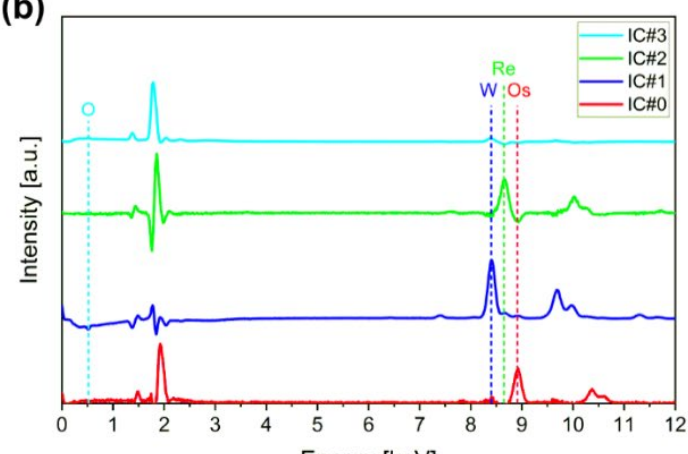

Energy $[\mathrm{keV}]$

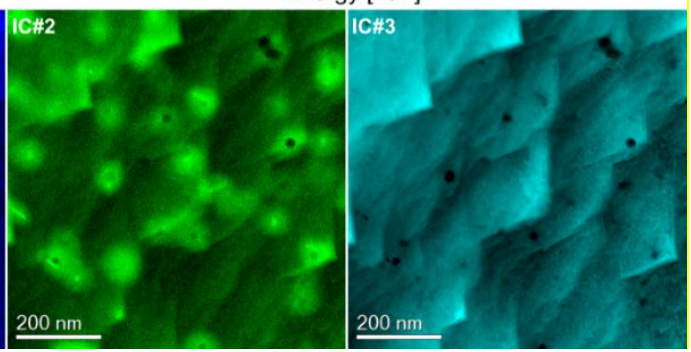

Figure 5: Results of a blind source separation (BSS) by PCA and ICA of the STEM-EDX data presented in Figure 6. (a) Screen plot of the first 30 principal components (the dashed line indicates the number of independent components). The inset shows a schematic cross-sectional view of the sample for illustration purposes. (b) Corresponding independent component spectra. (c) Independent component maps showing the presence of Os-rich cores (IC\#0), a W matrix (IC\#1), a Re-rich cloud around the Os-rich cores (IC\#2), and an oxide surface layer (IC\#3), which is most probably due to the sample preparation.

In order to understand the phases and their distribution in more detail, an ICA was performed on the STEM-EDX data presented in Figure 4. The results are shown Figure 5. Figure 5a illustrates that the first 4 principal components have a significantly larger variance than the remaining components. The inset shows a schematic representation of the sample that was derived by analyzing the individual independent component spectra (Figure 5b) and respective maps (Figure 7c). IC\#0 corresponds to the Os-rich precipitates that are surrounded by Re-rich clouds (IC\#2). Both are embedded in the $\mathrm{W}$ matrix (IC\#1). No significant elemental intermixture between the three elements was observed here. The fourth independent component was a thin oxide surface layer that originated most probably from sample preparation and could not be avoided.

The STEM-HAADF image in the top part of Figure 6a shows a representative sample region containing a $\mathrm{W}$ grain boundary (marked as GB in the image). In addition, it is evident from the image that in each $\mathrm{W}$ grain there is a denuded zone present that has a width of about $210 \mathrm{~nm}$. Further into the grain interior the situation is as described in Figure 6. Furthermore, it was found that in the center of the GB there is an enrichment of Os $(4.6 \mathrm{wt} \%)$ and $\operatorname{Re}(0.7 \mathrm{wt} \%)$ that has a width of about $30 \mathrm{~nm}$, which can be observed in the elemental maps presented in Figure $6 \mathrm{~b}-\mathrm{d}$. 

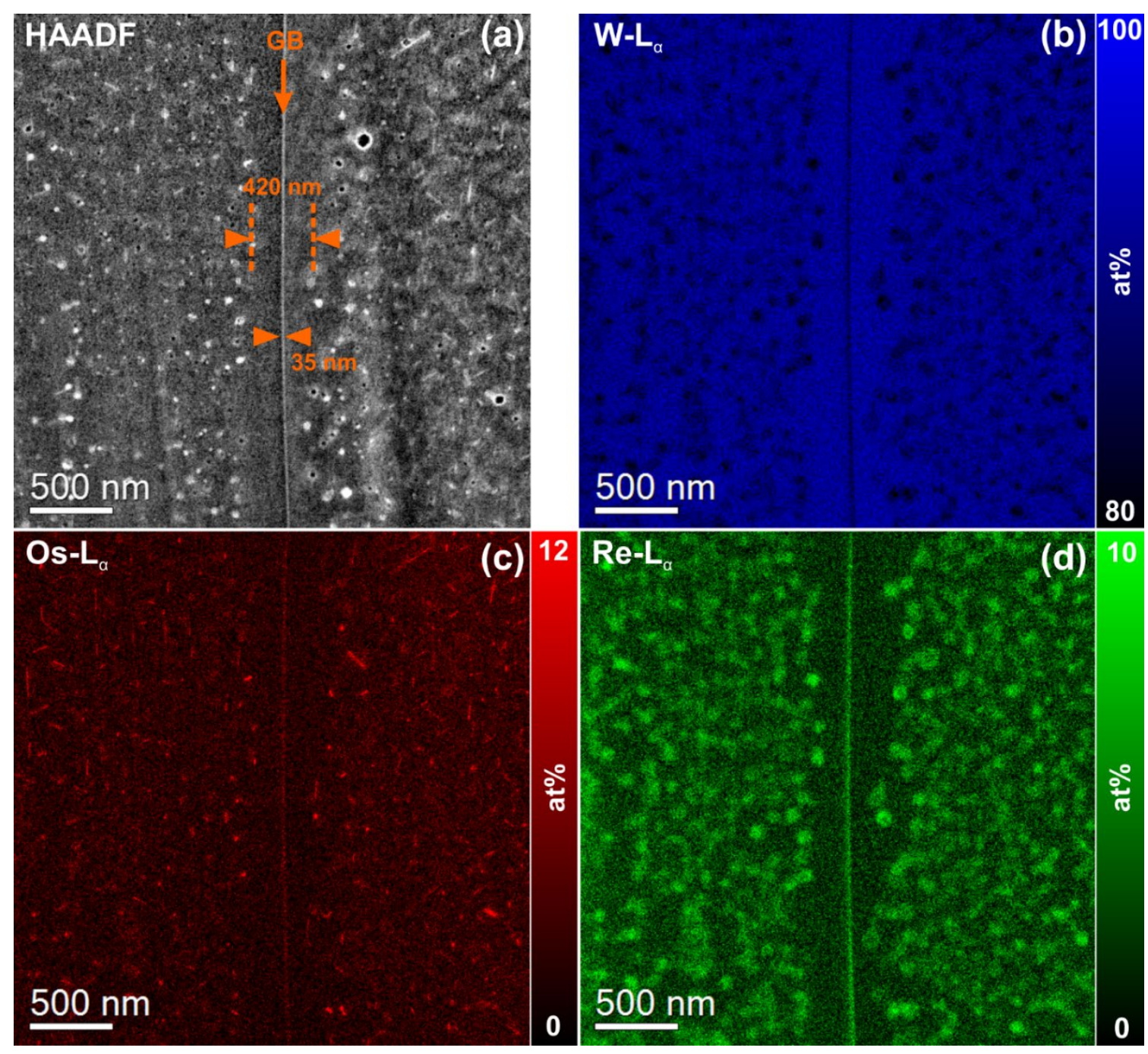

Figure 6: STEM-EDX mapping of a grain boundary. (a) STEM-HAADF image. The position of the W grain boundary (GB) is indicated in the HAADF image. Elemental maps of (b) W, (c) Os, and (d) Re display the location of elements with respect to the sample morphology.

\subsection{HRTEM analysis of precipitates}

High-resolution phase contrast images are acquired in order to further characterize the Os-rich precipitates as can be seen in Figure 7, Figure 8, and Figure 9. In these three figures, no structural difference between the pure W matrix and the Re-enriched cloud around the Os-rich precipitates are observed. Therefore, the region outside the Os-rich precipitates is labeled as W matrix in these figures. Figure 7a shows a high-resolution phase contrast image of a spherically shaped Os-rich phase embedded in the $\mathrm{W}$ matrix. The $\mathrm{W}$ matrix is positioned to have a [110] zone-axis orientation. Figure $7 \mathrm{~b}$ presents a magnified view of the region delimited by the orange square in (a). It is evident that the precipitate has the different crystalline structure compared to the matrix. However, without knowing the exact sample thickness the assignment of atomic positions in the precipitate is ambiguous due to the contrast reversals occurring for this particular imaging mode. Nevertheless, the fast Fourier transform (FFT) in Figure 7c calculated from Figure $7 \mathrm{a}$ sheds some light on the crystallographic structure of the precipitate and its crystallographic relationship to the $\mathrm{W}$ host matrix. The spots corresponding to the $\mathrm{W}$ matrix are indexed in red color and the ones originating from the precipitate are indexed in blue. For 
indexing the precipitate, we used the crystal structure of the WOs2 $\sigma$-phase (ICSD No. 150547), which is tetragonal. Furthermore, diffraction pattern calculations were carried out using the JEMS software (Figure 7d) in order to highlight the orientation relationship, i.e. (1-10)w $\|$ (1$12)_{\sigma},(002)_{\mathrm{W}} \|(-441)_{\sigma}$ and $[110]_{\mathrm{W}} \|[110]_{\sigma}$.
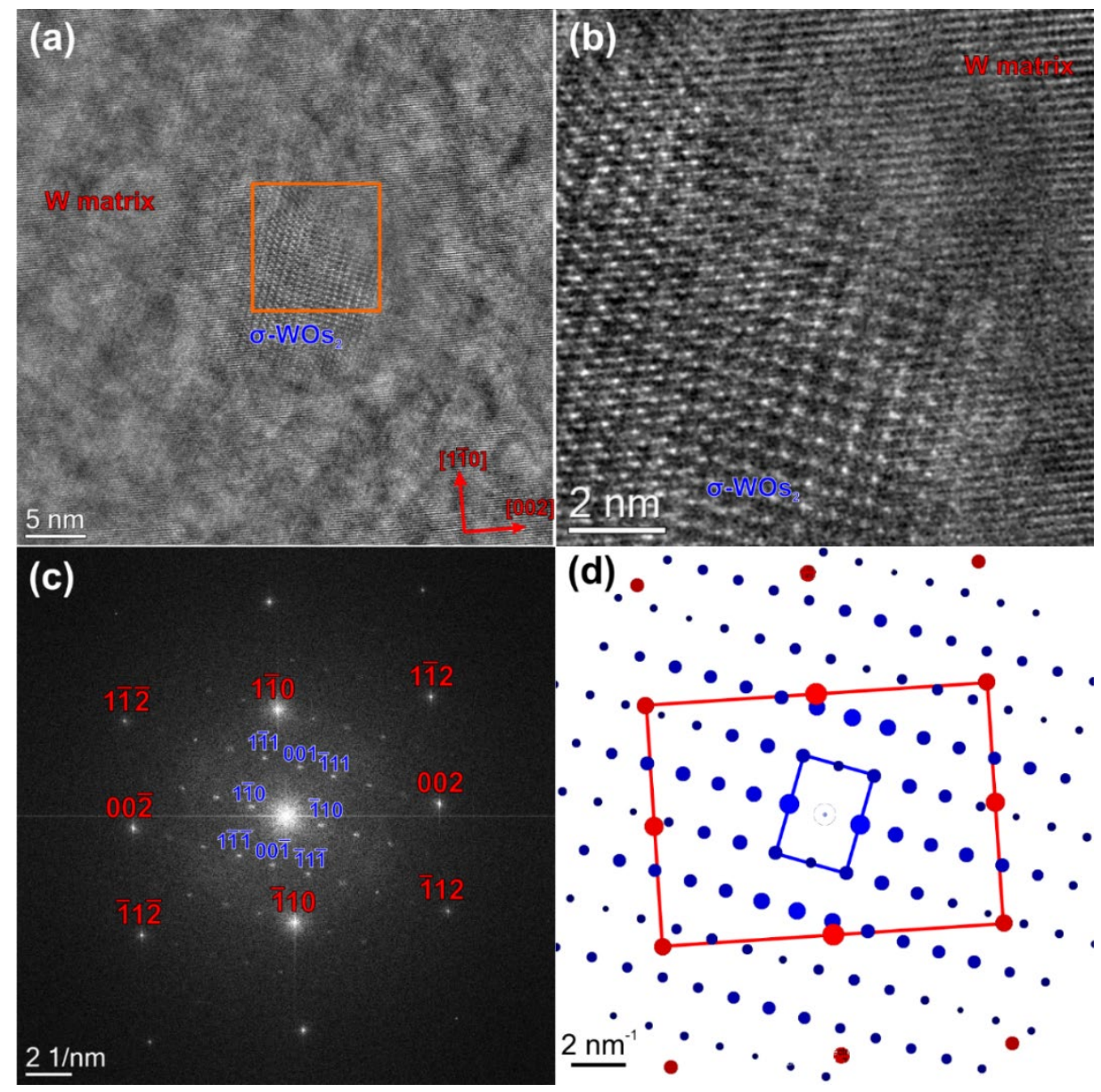

Figure 7: (a) High-resolution phase contrast image of a spherical-shaped Os-rich precipitate, which is identified as the WOs2 $\sigma$-phase. Magnified view (b) and diffractogram (FFT) (c) of the region delimited by the orange square in (a). (d) Simulated model of the diffractogram.

Figure 8a shows a high-resolution phase contrast image of a rod-shaped Os-rich precipitate, which is attached to a void. Figure $8 \mathrm{~b}$ is a magnified view of the region delimited by the orange square in (a). The situation is less evident here than in Figure 7, because there is only a slight change of the contrast. However, the diffractogram in Figure 8c reveals the presence of two different crystal lattices, i.e. the matrix oriented in [110] zone-axis (red indices) and the precipitate (blue indices). For indexing the precipitate, we used the crystalline structure of the $\mathrm{WRe}_{3} \chi$-phase as model structure (ICSD No. 650196) since no structure of a W-Os $\chi$-phase is reported in the ICSD database. The WRe $3 \chi$-phase has a body-centered cubic structure just as W. We therefore just replaced Re by Os, which should not remarkably affect the structure since the atomic radii of these atoms differ only by a few picometers ${ }^{14}$ and carried out diffraction pattern simulations by the JEMS software ${ }^{13}$. However, to provide more accurate results, the structure needs to be relaxed e.g. by applying density functional methods. The result of our simulation is shown in Figure $8 \mathrm{~d}$. The orientation relationship in this case was determined as $(1-10)_{\mathrm{W}}\left\|(2-40)_{\chi},(002)_{\mathrm{w}}\right\|(-5-32)_{\chi}$ and [110]w $\|[214]_{\chi}$. 

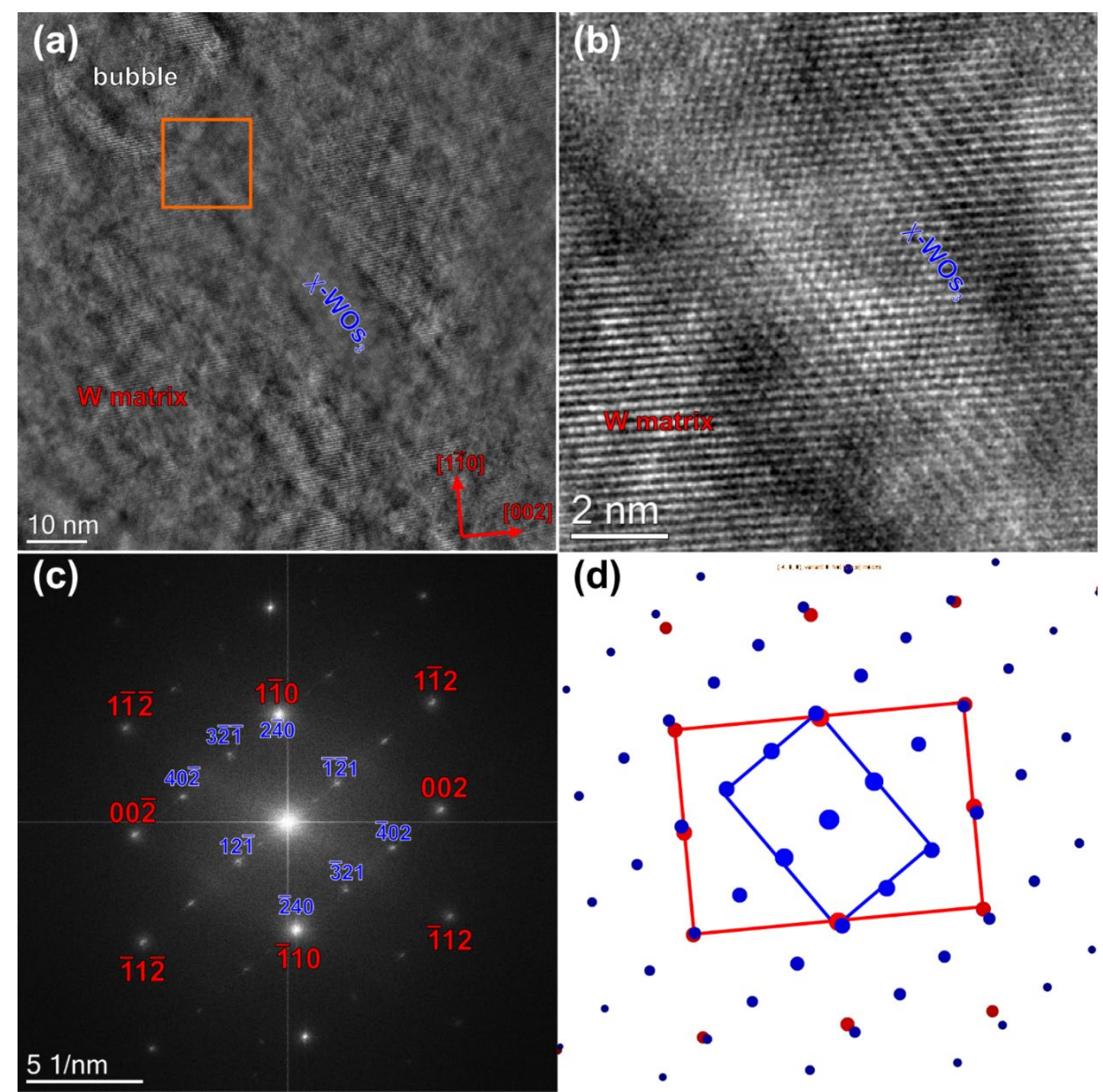

Figure 8: (a) High-resolution phase contrast image of a rod-shaped Os-rich precipitate, which is identified as the WOs 3 -Phase. Magnified view (b) and diffractogram (FFT) (c) of the region delimited by the orange square in (a). Simulated model of the diffractogram (d).

Figure 9a shows a rod-shaped Os-rich precipitate attached to a void of about $75 \mathrm{~nm}$ in size. The void surface is facetted, i.e. $\{110\},\{002\}$, and $\{112\}$-type facets can be observed. The Os-rich precipitate is attached to a $\{110\}$-type facet and seems to grow along a $\langle 112\rangle_{W}$-type direction. However, as can be seen in Figure 9b, there is Moiré contrast present that indicates that the lattice of the precipitate is shifted, rotated or both with respect to the matrix lattice. The Moiré fringes have a distance of about $1.9 \mathrm{~nm}$, which is also reflected in the diffractogram (see inset). Furthermore, the diffractogram suggests that the precipitate is a $\chi$-type because the $\{111\}$-type planes seem to be aligned, since the distance of $(111)_{\chi}$ is about 1/3 of (111)w. Careful analyses of Figure 9a points that the precipitate is inclined with respect to the image plane, i.e. clear Moiré contrast in the upper part vs. no Moiré contrast in the lower part (close to the void) of the precipitate. Figure $9 \mathrm{~d}$ presents a composite image that shows the elemental distribution of Os in red, the Re distribution in green and the $\mathrm{W}$ distribution in blue colours. 


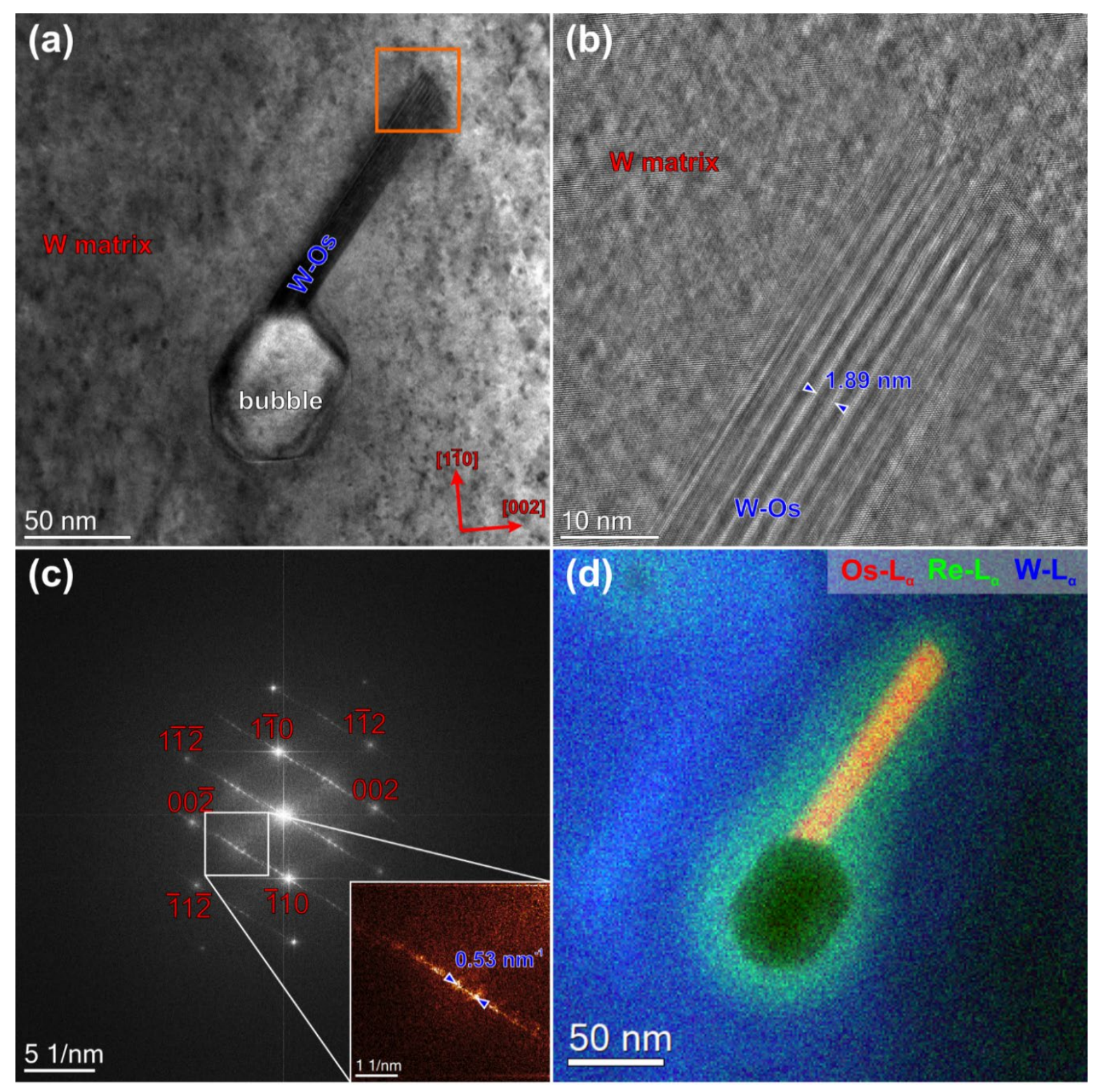

Figure 9: (a) TEM bright-field image of a faceted cavity attached to a W-Os rod. (b) Highresolution phase contrast image of the W-Os phase exhibiting Moiré fringes in the Os-rich part. (c) Diffractogram (FFT) of (b). (d) Composite STEM-EDX elemental map of the same sample region. 

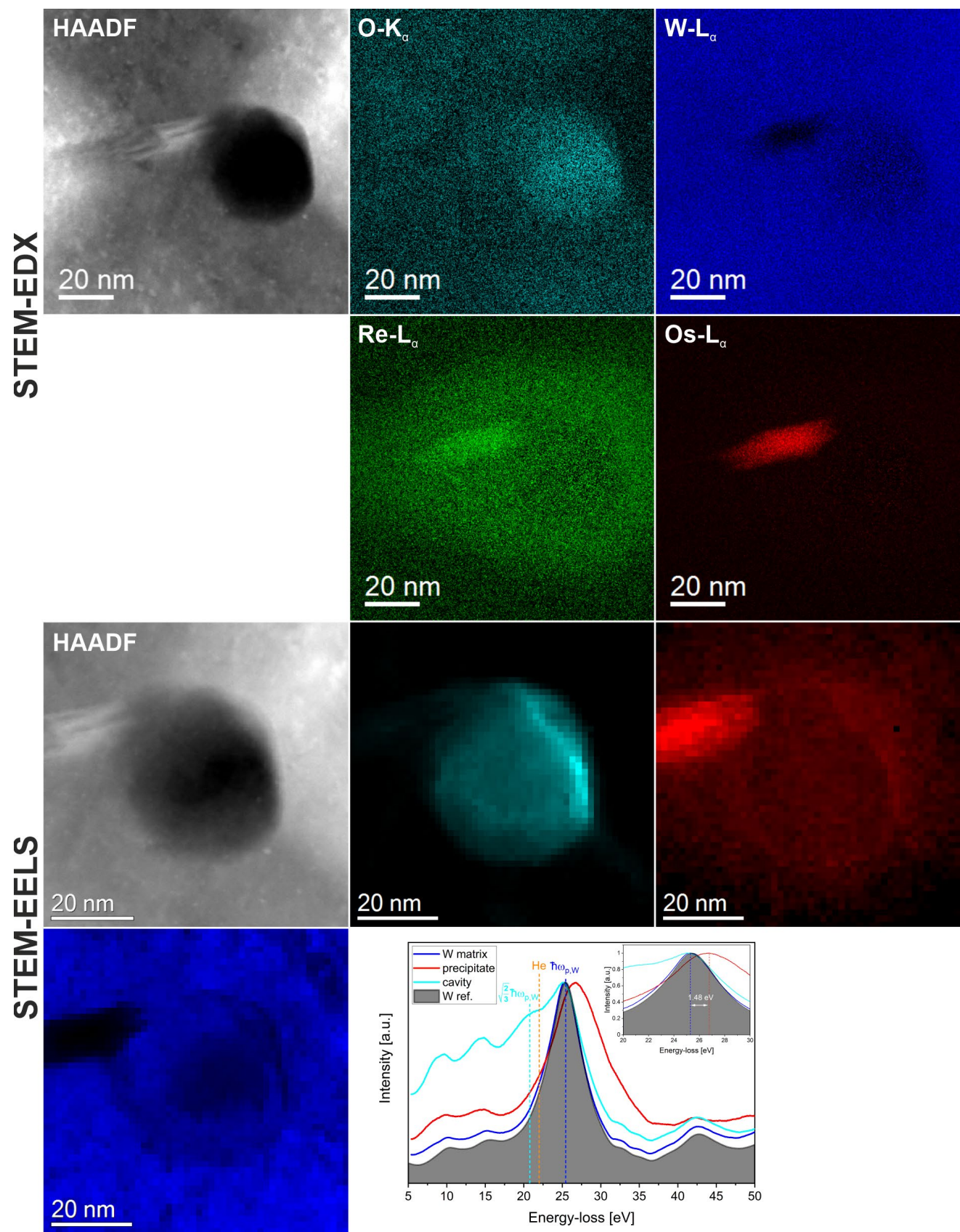

Figure 10: Combined STEM-EDX and low-loss STEM-EELS measurement of a individual cavity. The intensities of the STEM-EELS maps was obtained by NLLS fitting of the peaks located in the W plasmon peak area.

Figure 10 presents a combined STEM-EDX/STEM-EELS measurements performed for of a individual void. The STEM-EDX in the upper part shows that the void is attached to a Re-Osrich precipitate and is also surrounded by a Re-rich cloud. In addition, oxygen was fund to be present within the cavity. The STEM-EELS measurements presented in the lower part are performed to determine whether He is present in the cavity or not. An analysis of the acquired low-loss EELS data (shown on the right side in the lowest row) revealed that the bulk W spectrum is similar to a spectrum acquired from an unirradiated $\mathrm{W}$ sheet. Furthermore, it shows that the plasmon peak of the precipitate is shifted to higher energy-losses by $1.48 \mathrm{eV}$ with 
respect to the $\mathrm{W}$ matrix one. An additional peak (indicated by the orange dashed line) is present at an energy loss of about $20-21 \mathrm{eV}$ in the void area, the energy corresponding to the position of the He edge.

\section{Discussion}

The radiation-induced dislocation loops or defects, which are visible as structureless black dots a few nanometers in size, have an average size of $5 \mathrm{~nm}$ and a number density of $410^{23} \mathrm{~m}^{-3}$ (Figure 3, Table 3). Detailed information about the quantitative parameters of these defects and an understanding of the nature of these defects are necessary to explain e.g. radiation-induced embrittlement. As has been shown in several studies, the size and number density of dislocation loops do not vary significantly over a wide range of irradiation temperatures and damage doses $^{8,21,22}$. The average size of $5 \mathrm{~nm}$ is consistent with previously reported data for neutronirradiated tungsten, where it typically ranges from $1.2 \mathrm{~nm}$ to $8.0 \mathrm{~nm} .^{8}$ The formation of larger loops has rarely been reported in the scientific literature, e.g. Williams et. ${ }^{23}$ showed the formation of $150 \mathrm{~nm}$ loops in a W-Re alloy after neutron irradiation at $1500^{\circ} \mathrm{C}$. The measured number density is considerably higher compared to the literature, where its value varies between $110^{22} \mathrm{~m}^{-3}$ and $110^{23} \mathrm{~m}^{-3}$. This could reflect successful flash polishing procedure, which allows one an accurate detection of $\sim 1-2 \mathrm{~nm}$ loops.

The dislocation loops were observed in neutron irradiated $\mathrm{W}$ at an irradiation dose of $0.03 \mathrm{dpa} /$ $90^{\circ} \mathrm{C}^{8}$ and usually appear up to $600^{\circ} \mathrm{C}$ irradiation temperatures ${ }^{11,22,24,25}$. However, two works, which are consistent with our results, showed its presence up to $800^{\circ} \mathrm{C}^{8,25}$. The recent temperature-damage diagrams covers the irradiation temperature range from $100^{\circ} \mathrm{C}$ to $900^{\circ} \mathrm{C}$ and damage doses of up to $2.0 \mathrm{dpa}^{26}$. The damage structures were classified into three types: "loops", "loops and voids" and "precipitates and voids". It shows that at temperatures exceeding $500^{\circ} \mathrm{C}$ dislocation loops occur in combination with voids for doses below 0.6-0.7 dpa, whereas for doses larger than $1.0 \mathrm{dpa}$ the formation of loops was not reported. The critical aspect is that the diagram is based on a limited number of measurements, suggesting that the areas of the sections are not well defined and the occurrence of damage may deviate. In particular, this explains the deviation from our results concerning dislocation loops which show their existence at $1.25 \mathrm{dpa} / 800^{\circ} \mathrm{C}$. This indicates that the areas for the formation of dislocation loops should be modified.

In general, the observation of dislocation loops with TEM is a challenging task. By far not all publications contain the micrographs on which the loops could be identified. Due to the radioactive nature and small dimensions, most of TEM specimens have to be prepared by FIB, which itself causes severe surface damage hindering a doubtless identification of the loops. To remove the damaged layer, a low-energy ion cleaning or flash polishing have to be used. This procedure, however, is not successful in all cases so that the detection of small-sized dislocation loops $(<5 \mathrm{~nm})$ remains a challenge for an accurate quantification.

Burgers vector analysis of dislocation loops have only been reported for ion irradiated materials. In most cases the formation the loops in $\mathrm{W}$ show $\mathrm{b}^{1 / 2}<111>$ Burgers vector ${ }^{27-29}$, however, the presence of a small amount $(4 \%-6 \%)$ of $b<100>$ loops was reported ${ }^{30}$. These results are consistent with our analysis, which shows that $85 \%$ of the loops have a $b^{1} / 2<111>$ Burgers vector 
and only a small fraction of $b<100>$ loops was observed. The results of extensive dislocation loop analyses are summarized in ${ }^{31}$ and showed a marked difference between ion and neutron irradiation results regarding average size and number density. In addition, different irradiation conditions and different $\mathrm{W}$ to $\mathrm{Re}$ transmutation rates hamper a direct comparison of microstructure from different irradiation campaign.

Radiation induced void formation is the main damage process in $\mathrm{W}$. The presence of voids was reported in every specimen irradiated at temperatures exceeding $500^{\circ} \mathrm{C}{ }^{26,32}$. The formation starts at slightly higher irradiation doses as the dislocation loop formation, i.e. $0.15 \mathrm{dpa}{ }^{32}$. Increasing the dose increases the average void size and decreases their number density. Despite the fact that the voids were formed under irradiation conditions that varied over a wide range, their morphology is remarkably similar. The average size typically varies from $2 \mathrm{~nm}$ to $8 \mathrm{~nm}$

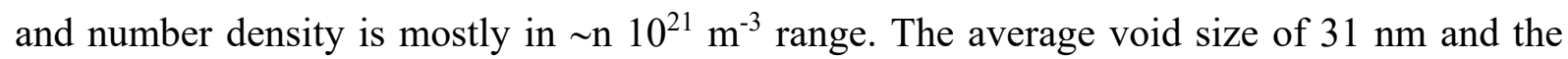
number density $410^{20} \mathrm{~m}^{-3}$ reported in the present work for the grain interior (Table 3 ) are both different from those previously reported ${ }^{4,6,32-34}$. The voids found at the temperatures ranging from $700^{\circ} \mathrm{C}$ to $900^{\circ} \mathrm{C}$ and doses exceeding 1.0 dpa are always smaller than $10 \mathrm{~nm}$, with an average size ranging from $3 \mathrm{~nm}$ to $7 \mathrm{~nm}$. The corresponding number density of voids in the same temperature and dose range lies between $210^{21}-110^{22} \mathrm{~m}^{-3}$. Larger number densities, i.e. larger than $110^{23} \mathrm{~m}^{-3}$, only occur for irradiation temperatures below $760^{\circ} \mathrm{C}$ at low doses ${ }^{25}$. The results summarized from different publications exhibit the tendency that the average void size increases from $4 \mathrm{~nm}$ to $7 \mathrm{~nm}$ and decrease in number density for doses exceeding $1.0 \mathrm{dpa}$, while the temperature influence on both parameters is statistically insignificant ${ }^{35}$. The voids formed at similar conditions $\left(\mathrm{T}_{\text {irr }}=900^{\circ} \mathrm{C}, 1.54 \mathrm{dpa}\right)$ are considerably smaller in size and have an order of magnitude higher number density than the voids reported in current work ${ }^{6}$. Thus, our results are not in line with previously published data and the difference is larger than that being expected if only damage and temperature are being considered.

We suggest that such differences in void morphologies could be explained by microstructural responses to neutron irradiation in different reactors. It was reported previously that the damage dose influences the void morphology to a significantly higher degree than the irradiation temperature. For example the presence of Re had a strong influence on the size and distribution of voids ${ }^{4,32}$. The accumulation of Re in $\mathrm{W}$ during neutron irradiation restrains their nucleation and growth. Moreover, the presence of Re and Os inside irradiated W can inhibit the growth of radiation-induced defects, whereas Os plays even more significant role than $\operatorname{Re}^{4,36}$. Thus, the concentration of transmutation products depends not only on irradiation temperature and dose, but also on the transmutation rates, which themselves depend on the actual neutron spectrum.

When analyzing the results of $\mathrm{W}$ irradiation in different reactors, significant differences in the size and distribution of the voids can be found. For example, voids in pure $\mathrm{W}$ irradiated in the JOYO fast reactor show a number density in the $10^{23} \mathrm{~m}^{-3}$ range. Fukuda et al. ${ }^{21}$ shows for example that their number density in pure $\mathrm{W}$ irradiated at $537^{\circ} \mathrm{C}$ comprises $1.910^{23} \mathrm{~m}^{-3}$. A similar values of $1.210^{23} \mathrm{~m}^{-3}\left(750^{\circ} \mathrm{C} / 1.54 \mathrm{dpa}\right)$ and $510^{23} \mathrm{~m}^{-3}(538 / 0.9 \mathrm{dpa})$ was reported by Tanno et al. ${ }^{11,37}$. The neutron spectrum in the JOYO reactor has a low cross-section for the transmutation of $\mathrm{W}$ and thus the changes in the chemical composition after irradiation are negligible. 
Both the HFIR and JMTR reactors provide a mixed neutron spectrum, which leads to an increased production of transmutation elements and consequently to a decrease of number density of voids by a factor of 10-150. Typically, the number density of voids in $\mathrm{W}$ irradiated in the range of $724^{\circ} \mathrm{C}$ to $764^{\circ} \mathrm{C}$ varied from $710^{20} \mathrm{~m}^{-3}$ to $110^{22} \mathrm{~m}^{-3}{ }^{25}$. The number density of voids in W-Re-alloys irradiated under similar conditions does not depend on the reactor type and is in the identical range from $310^{20} \mathrm{~m}^{-3}$ to $610^{21} \mathrm{~m}^{-3} 37$. According to Herschitz and Seidman 38 the growth of voids in W-Re alloys is affected by the recombination of point defects, i.e. vacancies and interstitials at a biased sink, e.g. dislocations. A similar argumentation is also valid for the generation of precipitates. They also claim that coherent precipitates in W-Re alloys are only generated if they are located close to damage cascades by primary knock-on atoms.

Table 3: Quantitative and statistical data of voids obtained near the grain boundaries separately for different zones

\begin{tabular}{|c|c|c|c|c|c|c|c|}
\hline $\begin{array}{l}\text { numbered } \\
\text { in Figure } 1\end{array}$ & designation & $\begin{array}{c}\text { location to } \\
\text { GB /nm }\end{array}$ & $\begin{array}{c}\text { void } \\
\text { number } \\
\text { density } \\
/ \times 10^{20} \mathbf{m}^{-3}\end{array}$ & $\begin{array}{c}\text { average } \\
\text { void size } \\
\text { /nm }\end{array}$ & $\begin{array}{c}\text { void } \\
\text { swelling } \\
/ \%\end{array}$ & $\begin{array}{c}\text { Averalge } \\
\text { loop size } \\
\text { /nm }\end{array}$ & $\begin{array}{c}\text { loop } \\
\text { number } \\
\text { density } \\
/ \times 10^{23} \mathrm{~m}\end{array}$ \\
\hline 1 & $\begin{array}{c}\text { void and } \\
\text { precipitates } \\
\text { denuded zone }\end{array}$ & $\begin{array}{l}\text { from GB to } \\
200 \mathrm{~nm}\end{array}$ & -- & -- & -- & & \\
\hline 2 & $\begin{array}{l}\text { void peak } \\
\text { zone }\end{array}$ & $\begin{array}{c}\text { from } 200 \\
\text { nm to } 500 \\
n m\end{array}$ & $8.0 \pm 2.5$ & 24 & $\sim 0.004$ & & \\
\hline 3 & $\begin{array}{c}\text { second } \\
\text { denuded zone }\end{array}$ & $\begin{array}{c}\text { from } 500 \\
\mathrm{~nm} \text { to } 1 \mu \mathrm{m}\end{array}$ & $1.9 \pm 0.5$ & 22 & -- & & \\
\hline-- & grain interior & $>1 \mu \mathrm{m}$ & $4.3 \pm 1.2$ & 31 & $\sim 0.02$ & $5.2 \pm 1$ & $4.1 \pm 1.5$ \\
\hline
\end{tabular}

Precipitates containing Re and Os are also formed in pure $\mathrm{W}$ irradiated in HFIR since the nuclear transmutation rate in HFIR is higher than in sodium-cooled reactors (JOYO) due to the high flux of thermal neutrons. Material analysed in our work shows the presence of $2 \% \operatorname{Re}$ and $0.2 \%$ Os after irradiation to $1.25 \mathrm{dpa}$. The transmutation rate of $\sim 1.6 \% \mathrm{Re} / \mathrm{dpa}$ is similar to that calculated for HFIR reactors by Greenwood and Garner ${ }^{39}$. The formation of precipitates with defined stoichiometric composition and crystalline structure starts when the irradiation temperatures exceed $500^{\circ} \mathrm{C}$ and leads to the formation of $\sigma-\mathrm{WRe}_{2}$ and $\chi-\mathrm{WRe}_{3}$ phases ${ }^{7,37}$. In particular, it was reported that the $\chi$-phase has a needle-like shape and the $\sigma$-phase has a spherical shape ${ }^{11,24}$.

Our investigations show the formation of Os-based $\sigma$ - and $\chi$-phases located in the center of the Re- and Os-rich clouds (see Figure 4 and Figure 5). The similar clouds also form around all voids, are not clearly recognizable in standard TEM or STEM images and can only be visualized in STEM-EDX element maps (see e.g. Figure 4). Recently formation of clouds with a diameter of about $\sim 30 \mathrm{~nm}$ has been reported in several publications ${ }^{3,5,6,35,40}$. The value is twice as small as the average diameter of $60-80 \mathrm{~nm}$ measured in our work. The clouds occur probably in early 
irradiation stages and serve as precursors for the later formation of $\sigma$-WOs2 and the $\chi$-WOs 3 precipitates when the local concentration of Os has reached a certain value through the further transmutation of Re. The existence of the $\sigma$-WOs2 phase in the neutron irradiated W-Os alloy was reported by Tanno, T. et al. ${ }^{41}$, whereas the existence of $\chi$-WOs3 phases was not reported up to now. Here, we present the first experimental evidence of this phase. However, for an enhanced understanding of the precipitation behavior more experimental data would be desirable.

The binary phase diagrams of $\mathrm{W}$-Re and $\mathrm{W}-\mathrm{Os}$ indicate the presence of a $\sigma$-type phase at elevated temperatures. In case of W-Os this phase extends from about 65 at $\%$ to about 80 at $\%$ $\mathrm{W}$ for temperatures ranging from 1000 to $3000^{\circ} \mathrm{C}$. The existence of $\chi$-WOs3 phase was not reported since the phase diagram was not explored for all concentration ranges and temperatures. In case of $\sigma-\mathrm{WRe}_{2}$ the situation is more complicated: above $2000^{\circ} \mathrm{C}$ the $\sigma$-phase is present in the range of $30 \mathrm{at} \%$ to $56 \mathrm{at} \% \mathrm{~W}$, whereas below the range of existence shifts from 30 at $\%$ to about 35 at $\%$ at $1600^{\circ} \mathrm{C}$. In addition, the $\chi$-WOs3 phase exists up to about $2100^{\circ} \mathrm{C}$ in the $\mathrm{W}$ concentration range of $26-28$ at $\%$. In neutron-irradiated material, however, due to the irradiation-enhanced diffusion of impurities, secondary phases are not necessarily formed in accordance with the phase equilibrium rules.

Figure 7 and Figure 8 clearly illustrate that there is a textural (epitaxial) relationship between the W-matrix and both types of precipitates. However, as can be seen in Figure 9, the structure of the precipitation is not always well extinct. (Semi-)coherent precipitates were also observed by atom-probe measurements in W-Re alloys ${ }^{38}$. In this case, W-Re alloys with similar precipitation behavior as in the present case were already studied by several groups ${ }^{11,24,41-43}$, who reported circular-shaped $\sigma$-type and platelet-shaped $\chi$-type precipitates. This agrees also with the binary W-Re phase diagram. However, in our case pure W was subjected to neutron irradiation. The available literature is more limited than for the W-Re alloys ${ }^{8,10,35}$. However, recently published results of neutron irradiated pure W show a similar microstructure as our sample for similar neutron irradiation conditions, respectively ${ }^{35,40}$. The crystallographic structure of the intragranular precipitates is still under debate at present time. On the one hand, Katoh et al. ${ }^{35}$ claim that the platelet-shaped precipitates found in samples irradiated at elevated temperatures and doses around one dpa are inconsistent with the $\sigma$ - and the $\chi$-type phase. Edmondson et al. ${ }^{40}$ on the other hand come to the conclusion that the needle-like precipitates are of the $\sigma$-type. Moreover, they found a clear relationship of the $\mathrm{W}$ matrix with the precipitates, i.e. the precipitates lie along the (011) planes. They attributed this behavior to the dislocation loops originating from collision cascades, which act as trap for diffusing Os and Re atoms thereby seeding the precipitates. In contrast to Katoh et al. ${ }^{35}$ we found that both types of precipitates ( $\sigma$ - and the $\chi$-type) are present within our sample. We found that out-of-plane the $\sigma$-phase [110] axis is oriented along the [110] of W, whereas in-plane the orientation relation is $(1-10)_{\mathrm{W}} \|(1-12)_{\sigma}$ and $(002)_{\mathrm{W}} \|(-441)_{\sigma}$. Whether the precipitate has a rod or needle shape or another one is not evident from Figure 7. In case of the $\chi$-type precipitates the orientation relationship is more complicated than for the $\sigma$-type precipitates: Out-of-plane the $\mathrm{W}$ [110] direction is parallel to the [214] direction of the $\chi$-phase, whereas in-plane it is $(1-10) \mathrm{w} \|(2-$ $40)_{\chi},(002) \mathrm{w} \|(-5-32) \chi$. To our knowledge, such orientation relationships have not been reported before for this metallic system. However, in case of the $\chi$-type phase the orientation relation is not the same for all as discussed in the following paragraph. 
Katoh et al. ${ }^{35}$ found that some precipitates are associated with a void and some are not, which is in accordance with our observations. Furthermore, we found that the voids are often facetted with the majority of the facets having a $\{110\}$-type orientation. This can be explained by considering the surface energies of $\mathrm{W}$, which are lowest for $\{110\}$-type surfaces ${ }^{44}$. If voids are facetted and have a precipitate attached, the precipitate grows out of a $\{110\}$-type surface. This might be attributed to the origin of both which is related to damage cascades. Some of the WOs precipitates were found to be inclined with respect to the matrix lattice (see for example Figure 9). This behavior was also observed by Edmondson et al. ${ }^{40}$. Since the statistics of analyzed precipitates is limited using high-resolution phase contrast images, tKD or EBSD-like orientation mapping in the TEM can improve the statistics significantly regarding the textural relationship between matrix and precipitates.

Grain boundaries play an important role in the formation and coarsening of radiation-induced defects (Figure 1b, Figure 6). They act as sinks for all kinds of point defects, i.e. vacancies and interstitials as well as in our case Re and Os atoms, resulting in the formation of a $200 \mathrm{~nm}$ wide void- and precipitate-free zone (called the denuded zone) adjacent to the grain boundaries (see Figure 1b, Figure 6). Next to the denuded zone, a $300 \mathrm{~nm}$ wide void peak zone has formed, in which the voids have a reduced average size, while the number density of them is twice as high as in the grain interior (see Table 3). The formation of larger $\chi$ and $\sigma$ precipitates in the zone also indicates a higher concentration of Re and Os compared to the grain interior (Figure 6). According to Fukuda et.al ${ }^{24}$, the higher diffusivity of interstitials in comparison to that of vacancies promotes the formation of a void-peak zone in the immediate vicinity of the denuded zone.

Next to the void peak zone we observed the formation of a second denuded zone of about $~ 500$ $\mathrm{nm}$ wide with an obviously lower number density and size of voids and Os-rich precipitates if compared to the grain interior (Table 3). In this area especially Os- and Re-rich "clouds" are dominating structures. This indicates that the formation of $\sigma$ - and $\chi$-phases occurs only in "clouds" with sufficient Re and Os concentration, obviously reached inside grains or void denuded zones. The low local concentration of Re and Os suppresses their formation and growth. The influence of the grain boundary on the distribution of transmutation products and the formation of radiation-induced defects has already been reported in the past 3,6,24,34. However, the width of the denuded zone reported in these works is typically $\sim 20 \mathrm{~nm}$ - a value that is 10 times lower than what is measured in this study.

It is known that besides $\mathrm{Re}$ and $\mathrm{Os}$ also $\mathrm{H}$ and $\mathrm{He}$ are generated in appm amounts by transmutation of impurities in $\mathrm{W}$ and its alloys ${ }^{45,46}$. From Be it is well-known that both elements are retained within the material in the form of gas-filled bubbles ${ }^{47}$. The same effect might be also valid for $\mathrm{W}$, however, in an attenuated form. In $\mathrm{W}$, the bulk volume plasmon is located at $25.3 \mathrm{eV}$ according to our measurements, which is close to the He edge at $22 \mathrm{eV}^{48}$. The additional peak located only in the void area (STEM-EELS in Figure 10) lies in principle in the correct energy range of the He edge. However, since STEM-EDX also revealed the presence of oxygen within the void acting as dielectric medium another effect called plasmon resonance is also likely to be present. In case of nanometer-sized spherical voids this effect will generate additional intensity in the EEL spectrum located at $\sqrt{2 / 3} \omega_{B}$, where $\omega_{B}$ is the bulk plasmon frequency ${ }^{49}$. In case of $\mathrm{W}$ this evaluates to $20.7 \mathrm{eV}$, which fits better than the He edge to the 
observed void spectrum. If the voids contain pressurized He gas, a blue shift of the He-K edge towards the $\mathrm{W}$ bulk plasmon can be expected hampering the He detection in this particular case. Atom-probe measurements by Herschitz and Seidman ${ }^{38}$ did not reveal any He within voids, however, they found evidence of $\mathrm{He}$ associated with precipitates. The question of the $\mathrm{He}$ presence in the voids remains still open.

In general, experimental data collected in recent years shows that the microstructure of neutronirradiated $\mathrm{W}$ depends on the temperature and damage dose, as well as on the concentration of transmutation products. For the latter one the applied neutron spectrum is of great importance, which itself is different for each reactor type or shielding factor, e.g. position of the sample in the irradiation rig and of the rig itself in the reactor core. Our analyses form the basis for a detailed understanding of the microstructural development of $\mathrm{W}$ under neutron irradiation. However, considerably more research is needed to uncover the structural effects of a true fusion neutron spectrum.

\section{Conclusions}

This paper presents the results of extensive microstructural analyses of $\mathrm{W}$ samples irradiated at $800^{\circ} \mathrm{C}$ to $\sim 1.0 \mathrm{dpa}$. The formation of dislocation loops, voids and precipitates consisting of ReOs $\sigma$ - and $\chi$-phases was detected and characterized in detail. The results of the study can be summarized as follows:

- The dislocation loops have an average size of $5 \mathrm{~nm}$ and number density of $510^{23} \mathrm{~m}^{-3}$. About $95 \%$ of the loops have a $b^{1 / 2}<111>$ Burgers vector. These data are comparable with the data of $\mathrm{W}$ irradiated in the HFIR.

- The voids with an average size of $31 \mathrm{~nm}$ and number density of $410^{20} \mathrm{~m}^{-3}$ significantly different from the data being published in the literature. The average size is about 4-6 times larger and the number density is one order of magnitude lower than the published data for $\mathrm{W}$ irradiated at $\sim 800^{\circ} \mathrm{C}$.

- Near grain boundaries, we detected the formation of a $\sim 200 \mathrm{~nm}$ wide void denuded zone and $\mathrm{a} \sim 300 \mathrm{~nm}$ wide void peak zone.

- The Os induced by the transmutation has formed $\sigma$-WOs2 and $\chi$-WOs3 precipitates with spherical and needle-like shape, respectively.

- The $\chi$-WOs3 phase is often associated with a void in contrast to the $\sigma$-WOs2.

- The coherent precipitation of both $\sigma$ and $\chi$ is phases was determined. The [110] direction of $\sigma$-phase is oriented along that [110] of $\mathrm{W}$, whereas in-plane the orientation relation is $(1-10)_{\mathrm{W}} \|(1-12)_{\sigma}$ and $(002)_{\mathrm{W}} \|(-441)_{\sigma}$. The out-of-plane the $\mathrm{W}$ [110] direction is parallel to the [214] direction of the $\chi$-type precipitates, whereas in-plane it is $(1-10)_{\mathrm{W}} \|$ $(2-40)_{\chi},(002) \mathrm{w} \|(-5-32)_{\chi}$.

- Formation of Re and Os rich clouds around voids and precipitates was observed and analyzed. 


\section{Acknowledgements}

This work has been carried out within the framework of the EUROfusion Consortium and has received funding from the Euratom research and training programme 2019-2020 under grant agreement No 633053. The views and opinions expressed herein do not necessarily reflect those of the European Commission.

\section{References}

1. Bolt, H. et al. Materials for the plasma-facing components of fusion reactors. Journal of Nuclear Materials 329-333, 66-73; 10.1016/j.jnucmat.2004.04.005 (2004).

2. Rieth, M., Doerner, R., Hasegawa, A., Ueda, Y. \& Wirtz, M. Behavior of tungsten under irradiation and plasma interaction. Journal of Nuclear Materials 519, 334-368; 10.1016/j.jnucmat.2019.03.035 (2019).

3. Lloyd, M. J. et al. Decoration of voids with rhenium and osmium transmutation products in neutron irradiated single crystal tungsten. Scripta Materialia 173, 96-100; 10.1016/j.scriptamat.2019.07.036 (2019).

4. Hasegawa, A., Fukuda, M., Yabuuchi, K. \& Nogami, S. Neutron irradiation effects on the microstructural development of tungsten and tungsten alloys. Journal of Nuclear Materials 471, 175-183; 10.1016/j.jnucmat.2015.10.047 (2016).

5. Hwang, T. et al. Effect of neutron irradiation on rhenium cluster formation in tungsten and tungsten-rhenium alloys. Journal of Nuclear Materials 507, 78-86; 10.1016/j.jnucmat.2018.04.031 (2018).

6. Klimenkov, M. et al. Effect of neutron irradiation on the microstructure of tungsten. Nuclear Materials and Energy 9, 480-483; 10.1016/j.nme.2016.09.010 (2016).

7. Hasegawa, A., Fukuda, M., Nogami, S. \& Yabuuchi, K. Neutron irradiation effects on tungsten materials. Fusion Engineering and Design 89, 1568-1572; 10.1016/j.fusengdes.2014.04.035 (2014).

8. Koyanagi, T. et al. Microstructural evolution of pure tungsten neutron irradiated with a mixed energy spectrum. Journal of Nuclear Materials 490, 66-74; 10.1016/j.jnucmat.2017.04.010 (2017).

9. Fukuda, M., Hasegawa, A., Tanno, T., Nogami, S. \& Kurishita, H. Property change of advanced tungsten alloys due to neutron irradiation. Journal of Nuclear Materials 442, S273-S276; 10.1016/j.jnucmat.2013.03.058 (2013).

10. Hasegawa, A., Fukuda, M., Tanno, T. \& Nogami, S. Neutron Irradiation Behavior of Tungsten. Mater. Trans. 54, 466-471; 10.2320/matertrans.MG201208 (2013).

11. Tanno, T., Fukuda, M., Nogami, S. \& Hasegawa, A. Microstructure Development in Neutron Irradiated Tungsten Alloys. Mater. Trans. 52, 1447-1451; 10.2320/matertrans.MBW201025 (2011).

12. Hasanzadeh, S. et al. Three-dimensional scanning transmission electron microscopy of dislocation loops in tungsten. Micron (Oxford, England : 1993) 113, 24-33; 10.1016/j.micron.2018.05.010 (2018).

13. Stadelmann, P. A. EMS - a software package for electron diffraction analysis and HREM image simulation in materials science. Ultramicroscopy 21, 131-145; 10.1016/03043991(87)90080-5 (1987). 
14. Clementi, E., Raimondi, D. L. \& Reinhardt, W. P. Atomic Screening Constants from SCF Functions. II. Atoms with 37 to 86 Electrons. The Journal of Chemical Physics, 47(4), 1300-1307. The Journal of Chemical Physics 47, 1300-1307; 10.1063/1.1712084 (1967).

15. La Peña, F. D. et al. hyperspy/hyperspy: HyperSpy 1.6.0 (Zenodo, 2020).

16. Yin, C., Terentyev, D., Zhang, T., Petrov, R. H. \& Pardoen, T. Impact of neutron irradiation on the strength and ductility of pure and $\mathrm{ZrC}$ reinforced tungsten grades. Journal of Nuclear Materials 537, 152226; 10.1016/j.jnucmat.2020.152226 (2020).

17. Terentyev, D., Yin, C., Dubinko, A., Chang, C. C. \& You, J. H. Neutron irradiation hardening across ITER diverter tungsten armor. International Journal of Refractory Metals and Hard Materials, 105437; 10.1016/j.ijrmhm.2020.105437 (2020).

18. Klimenkov, M. et al. New insights into microstructure of irradiated beryllium based on experiments and computer simulations. Sci Rep 10; 10.1038/s41598-020-64654-5 (2020).

19. Yao, B., Edwards, D. J. \& Kurtz, R. J. TEM characterization of dislocation loops in irradiated bcc Fe-based steels. Journal of Nuclear Materials 434, 402-410; 10.1016/j.jnucmat.2012.12.002 (2013).

20. Prokhodtseva, A., Décamps, B., Ramar, A. \& Schäublin, R. Impact of He and Cr on defect accumulation in ion-irradiated ultrahigh-purity $\mathrm{Fe}(\mathrm{Cr})$ alloys. Acta Materialia 61, 69586971; 10.1016/j.actamat.2013.08.007 (2013).

21. Fukuda, M., Tanno, T., Nogami, S. \& Hasegawa, A. Effects of Re Content and Fabrication Process on Microstructural Changes and Hardening in Neutron Irradiated Tungsten. Mater. Trans. 53, 2145-2150; 10.2320/matertrans.MBW201110 (2012).

22. He, J. C., Hasegawa, A. \& Abe, K. Effects of transmutation elements on the defect structure development of $\mathrm{W}$ irradiated by protons and neutrons. Journal of Nuclear Materials 377, 348-351; 10.1016/j.jnucmat.2008.03.014 (2008).

23. Williams, R. K., Wiffen, F. W., Bentley, J. \& Stiegler, J. O. Irradiation induced precipitation in tungsten based, W-Re alloys. MTA 14, 655-666; 10.1007/BF02643781 (1983).

24. Fukuda, M., Yabuuchi, K., Nogami, S., Hasegawa, A. \& Tanaka, T. Microstructural development of tungsten and tungsten-rhenium alloys due to neutron irradiation in HFIR. Journal of Nuclear Materials 455, 460-463; 10.1016/j.jnucmat.2014.08.002 (2014).

$25 . \mathrm{Hu}, \mathrm{X}$. et al. Irradiation hardening of pure tungsten exposed to neutron irradiation. Journal of Nuclear Materials 480, 235-243; 10.1016/j.jnucmat.2016.08.024 (2016).

26. Marian, J. et al. Recent advances in modeling and simulation of the exposure and response of tungsten to fusion energy conditions. Nucl. Fusion 57, 92008; 10.1088/17414326/aa5e8d (2017).

27. Yi, X., Jenkins, M. L., Hattar, K., Edmondson, P. D. \& Roberts, S. G. Characterisation of radiation damage in $\mathrm{W}$ and $\mathrm{W}$-based alloys from $2 \mathrm{MeV}$ self-ion near-bulk implantations. Acta Materialia 92, 163-177; 10.1016/j.actamat.2015.04.015 (2015).

28. van Renterghem, W. \& Uytdenhouwen, I. Investigation of the combined effect of neutron irradiation and electron beam exposure on pure tungsten. Journal of Nuclear Materials 477, 77-84; 10.1016/j.jnucmat.2016.05.008 (2016).

29. Sikka, V. K. \& Moteff, J. "Rafting" in neutron irradiated tungsten. Journal of Nuclear Materials 46, 217-219; 10.1016/0022-3115(73)90139-6 (1973).

30. Harrison, R. W., Hinks, J. A. \& Donnelly, S. E. Influence of pre-implanted helium on dislocation loop type in tungsten under self-ion irradiation. Scripta Materialia 150, 61-65; 10.1016/j.scriptamat.2018.02.040 (2018).

31. Yi, X. et al. In situ study of self-ion irradiation damage in $\mathrm{W}$ and $\mathrm{W}-5 \mathrm{Re}$ at $500{ }^{\circ} \mathrm{C}$. Philosophical Magazine 93, 1715-1738; 10.1080/14786435.2012.754110 (2013).

32. He, J.C., Tang, G.Y., Hasegawa, A. \& Abe, K. Microstructural development and irradiation hardening of $\mathrm{W}$ and $\mathrm{W}-(3-26) \mathrm{wt} \% \mathrm{Re}$ alloys after high-temperature neutron irradiation to 0.15 dpa. Nucl. Fusion 46, 877-883; 10.1088/0029-5515/46/11/001 (2006). 
33. Garrison, L. M., Katoh, Y. \& Kumar, N.A.P. K. Mechanical properties of single-crystal tungsten irradiated in a mixed spectrum fission reactor. Journal of Nuclear Materials 518, 208-225; 10.1016/j.jnucmat.2019.02.050 (2019).

34. Hu, X. et al. Transmutation-induced precipitation in tungsten irradiated with a mixed energy neutron spectrum. Acta Materialia 165, 51-61; 10.1016/j.actamat.2018.11.032 (2019).

35. Katoh, Y. et al. Response of unalloyed tungsten to mixed spectrum neutrons. Journal of Nuclear Materials 520, 193-207; 10.1016/j.jnucmat.2019.03.045 (2019).

36. Tanno, T. et al. Effects of Transmutation Elements on Neutron Irradiation Hardening of Tungsten. Mater. Trans. 48, 2399-2402; 10.2320/matertrans.MAW200722 (2007).

37. Tanno, T. et al. Precipitation of Solid Transmutation Elements in Irradiated Tungsten Alloys. Mater. Trans. 49, 2259-2264; 10.2320/matertrans.MAW200821 (2008).

38. Herschitz, R. \& Seidman, D. N. Radiation-induced precipitation in fast-neutron irradiated tungsten-rhenium alloys: An atom-probe field-ion microscope study. Nuclear Instruments and Methods in Physics Research Section B: Beam Interactions with Materials and Atoms 7-8, 137-142; 10.1016/0168-583X(85)90544-0 (1985).

39. Greenwood, L. R. \& Garner, F. A. Transmutation of Mo, Re, W, Hf, and V in various irradiation test facilities and STARFIRE. Journal of Nuclear Materials 212-215, 635-639; 10.1016/0022-3115(94)90136-8 (1994).

40. Edmondson, P. D., Gault, B. \& Gilbert, M. R. An atom probe tomography and inventory calculation examination of second phase precipitates in neutron irradiated single crystal tungsten. Nucl. Fusion 60, 126013; 10.1088/1741-4326/abb149 (2020).

41. Tanno, T. et al. Effects of transmutation elements on the microstructural evolution and electrical resistivity of neutron-irradiated tungsten. Journal of Nuclear Materials 386-388, 218-221; 10.1016/j.jnucmat.2008.12.091 (2009).

42. Harrison, R. W. On the use of ion beams to emulate the neutron irradiation behaviour of tungsten. Vacuum 160, 355-370; 10.1016/j.vacuum.2018.11.050 (2019).

43. Nemoto, Y., Hasegawa, A., Satou, M. \& Abe, K. Microstructural development of neutron irradiated W-Re alloys. Journal of Nuclear Materials 283-287, 1144-1147; 10.1016/S0022-3115(00)00290-7 (2000).

44. Zhang, J.-M., Ma, F. \& Xu, K.-W. Calculation of the surface energy of bcc metals by using the modified embedded-atom method. Surf. Interface Anal. 35, 662-666; 10.1002/sia.1587 (2003).

45. Gilbert, M. R. \& Sublet, J.-C. Neutron-induced transmutation effects in W and W-alloys in a fusion environment. Nucl. Fusion 51, 43005; 10.1088/0029-5515/51/4/043005 (2011).

46. Sawan, M. E. Transmutation of Tungsten in Fusion and Fission Nuclear Environments. Fusion Science and Technology 66, 272-277; 10.13182/FST13-717 (2014).

47. Klimenkov, M. et al. First simultaneous detection of helium and tritium inside bubbles in beryllium. Micron (Oxford, England : 1993) 127, 102754; 10.1016/j.micron.2019.102754 (2019).

48. Ahn, C. C. Transmission electron energy loss spectrometry in materials science and the EELS atlas. 2nd ed. (Wiley-VCH, Weinheim Germany, 2004).

49. Prodan, E., Radloff, C., Halas, N. J. \& Nordlander, P. A hybridization model for the plasmon response of complex nanostructures. Science (New York, N.Y.) 302, 419-422; 10.1126/science.1089171 (2003). 
Author's contributions: M.D. performed HRTEM and the analytical investigation of precipitates and described these results in the manuscript. M.K. performed microstructural analysis of dislocation loops, voids, analyzed the structure near the grain boundary and described these results in the manuscript. U.J. performed targeted cutting of FIB lamellae from the cross sections for TEM analysis. M.R. together with $\mathrm{H}$ C.S. contributed to the data and literature analysis and interpretation of the results. D.T. organized and managed the neutron irradiation program. The authors do not declare competing interests. 


\section{Figures}

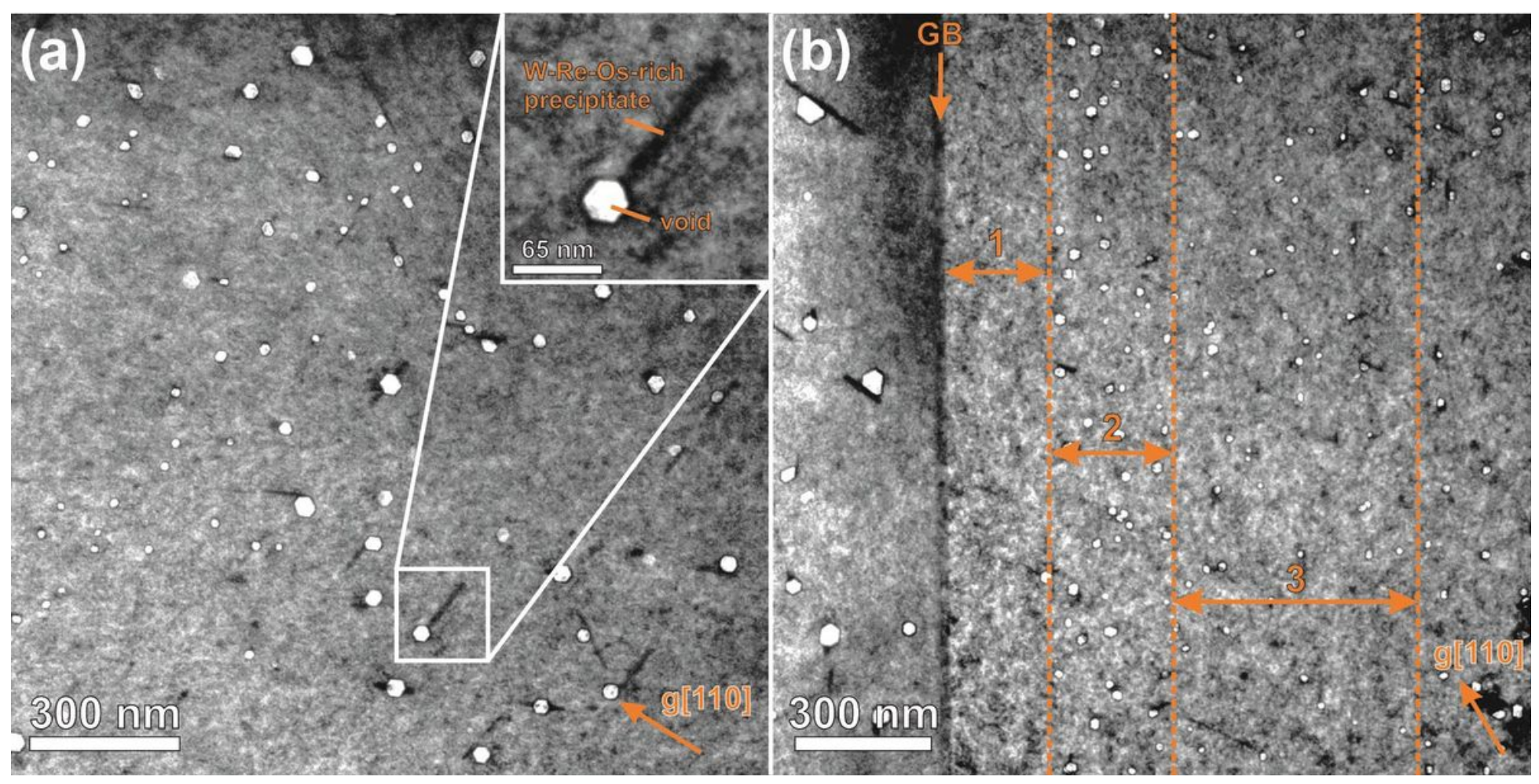

Figure 1

Bright-field TEM images acquired from neutron-irradiated W. (a) A representative area of a grain interior with voids (bright) and Re-Os-rich precipitates (dark strips). The inset shows a magnified view of a individual void attached to a W-Re-Os-rich precipitate. (b) A representative grain boundary (GB) region. The numbered areas are (1) the void denuded zone, (2) void-peak zone and (3) the second denuded zone. 

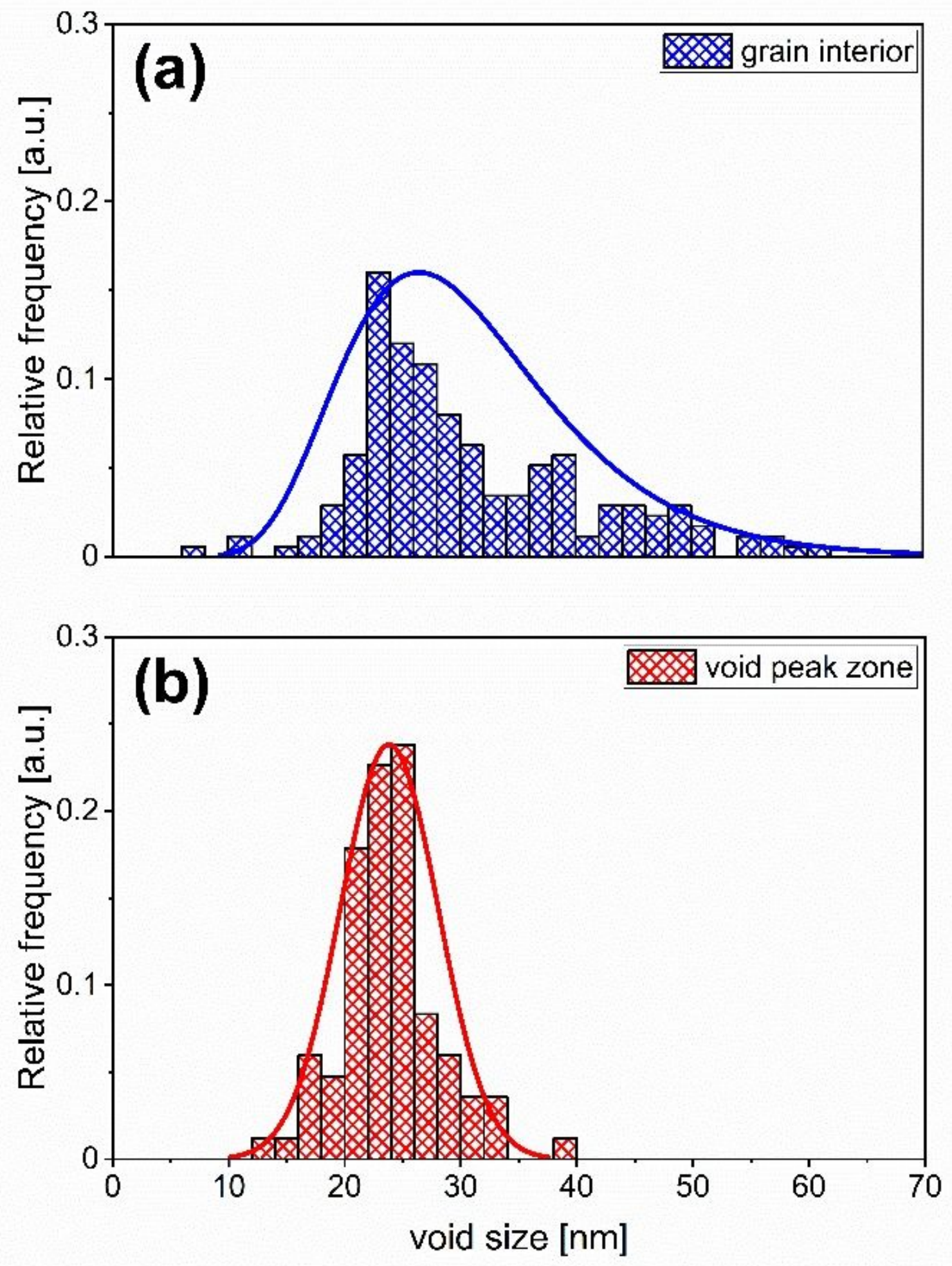

Figure 2

Size distribution histograms of the voids registered in the (a) grain interior and (b) void peak zone. 


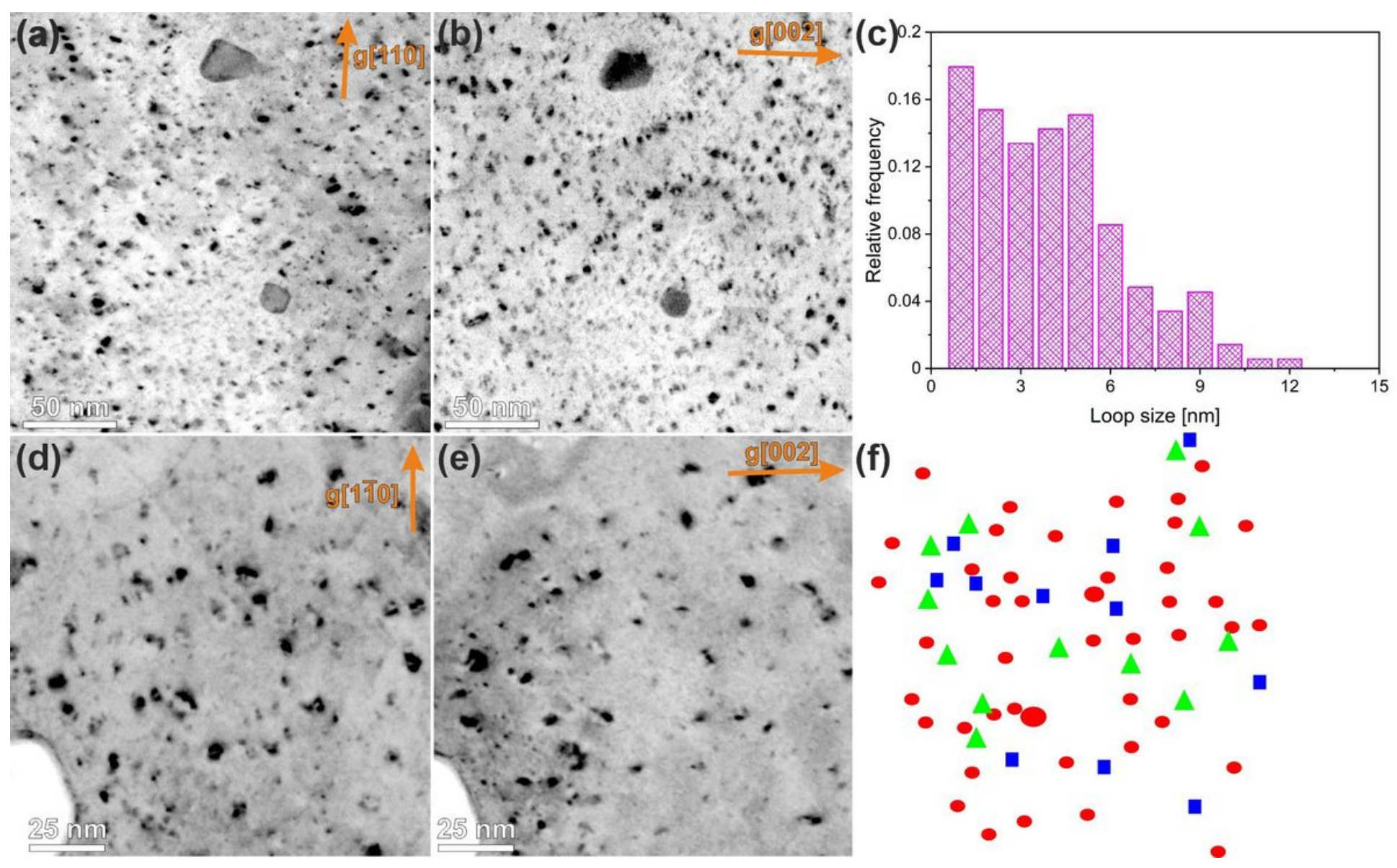

Figure 3

Visualization of dislocation loops and black dots in neutron-irradiated $\mathrm{W}$ resolved by reverse contrast dark-field (DF) imaging. DF images obtained with g[110] and g[002] g-vectors near the [110] zone-axis (a) and (b). These images were the basis for the loop size diagram (c). DF images obtained with g[110] and $g[002]$ g-vectors near the [110] zone-axis (d) and (e). The loops with $b 1 / 2 \otimes 111 \rrbracket$ and $b \otimes 100 \otimes$ Burgers vectors are imaged in the part (f) with red circles and blue squares, respectively. The loops with undefined Burgers vector are denoted by green triangles. 


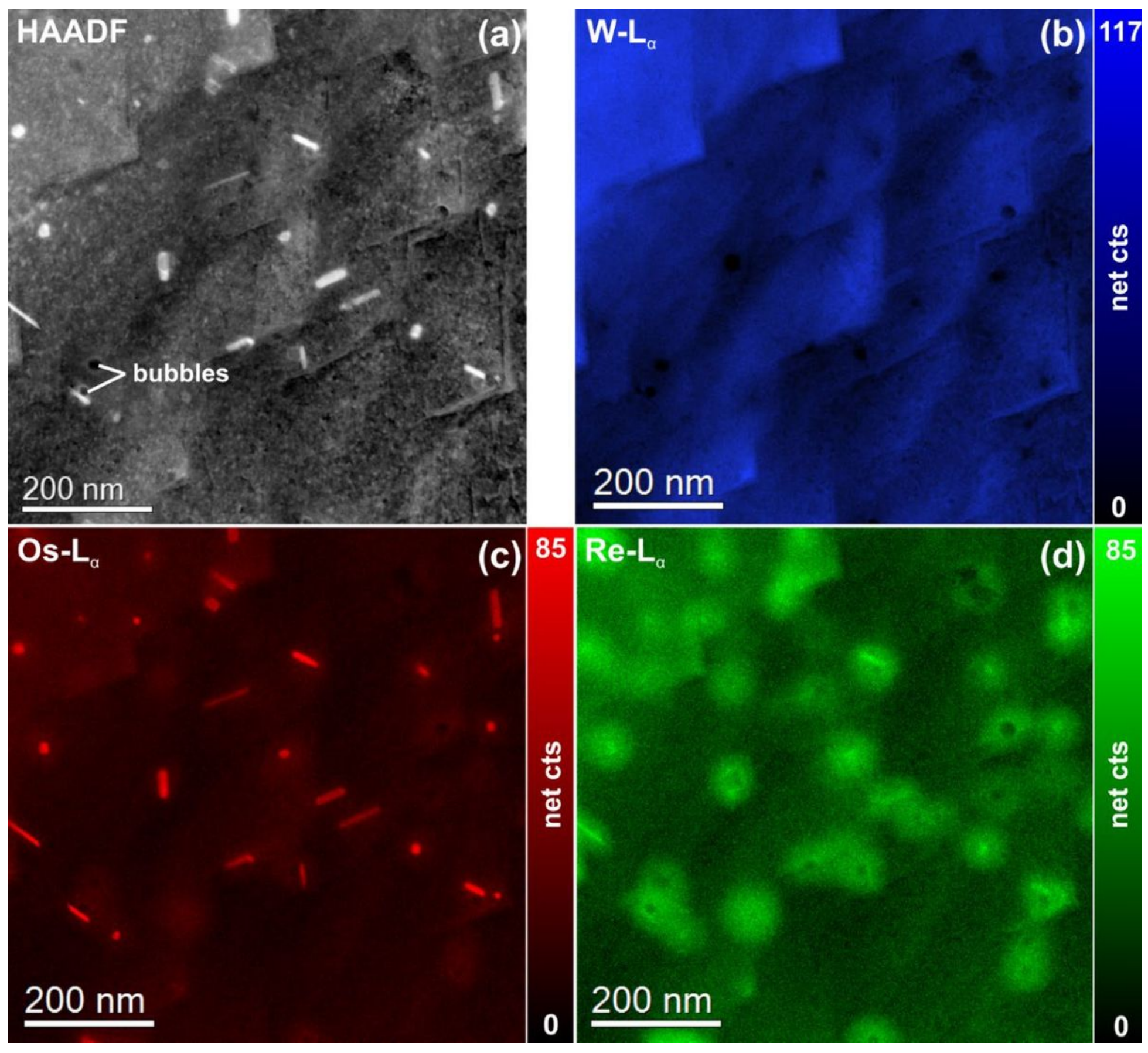

Figure 4

STEM-EDX spectrum image of a representative region in a neutron-irradiated W specimen. (a) STEMHAADF image. Elemental maps of (b) W, (c) Os, and (d) Re display the location of elements with respect to the sample morphology. The tile-like structure in the HAADF image and the W map is surface topography due to flash polishing. 
(a)

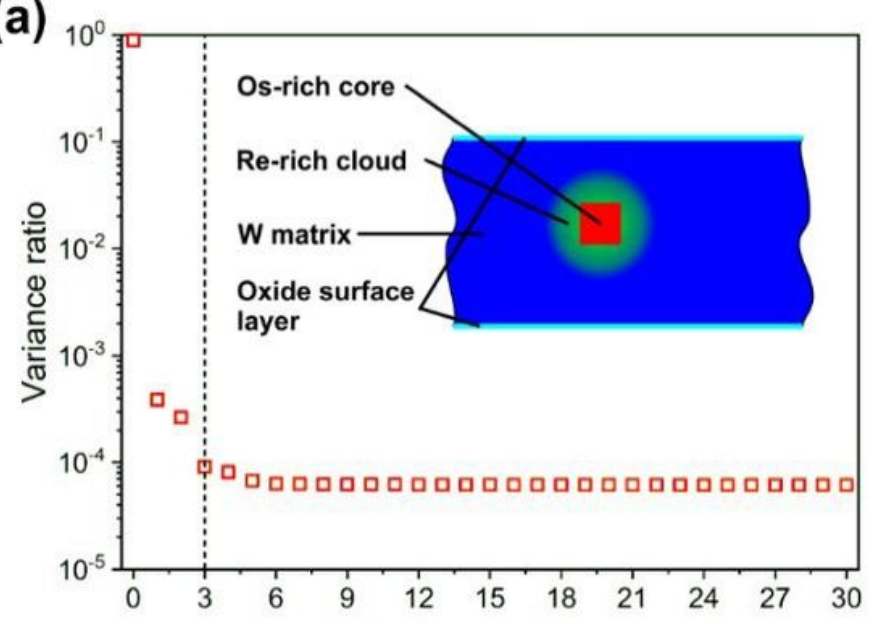

(c)

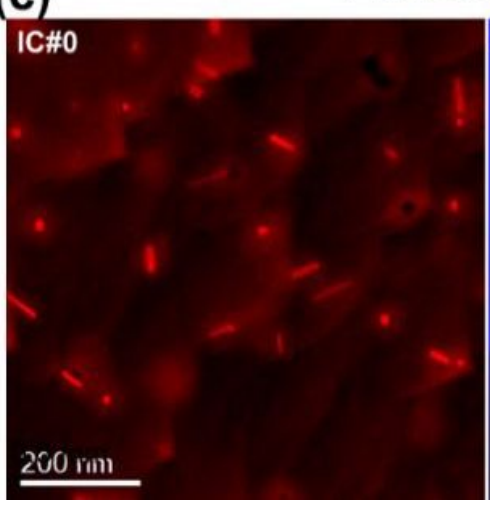

(b)
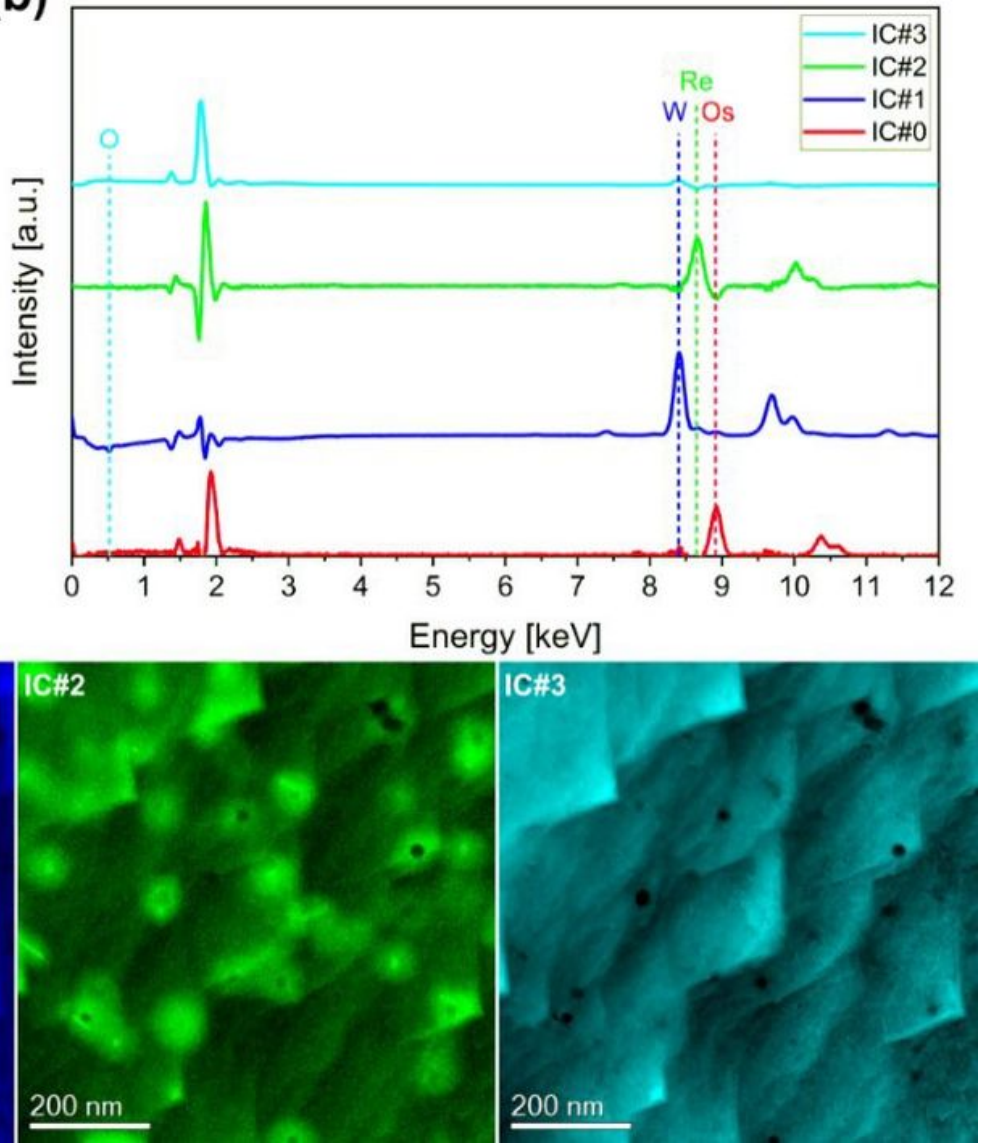

Figure 5

Results of a blind source separation (BSS) by PCA and ICA of the STEM-EDX data presented in Figure 6 . (a) Screen plot of the first 30 principal components (the dashed line indicates the number of independent components). The inset shows a schematic cross-sectional view of the sample for illustration purposes.

(b) Corresponding independent component spectra. (c) Independent component maps showing the presence of Os-rich cores (IC\#0), a W matrix (IC\#1), a Re-rich cloud around the Os-rich cores (IC\#2), and an oxide surface layer (IC\#3), which is most probably due to the sample preparation. 


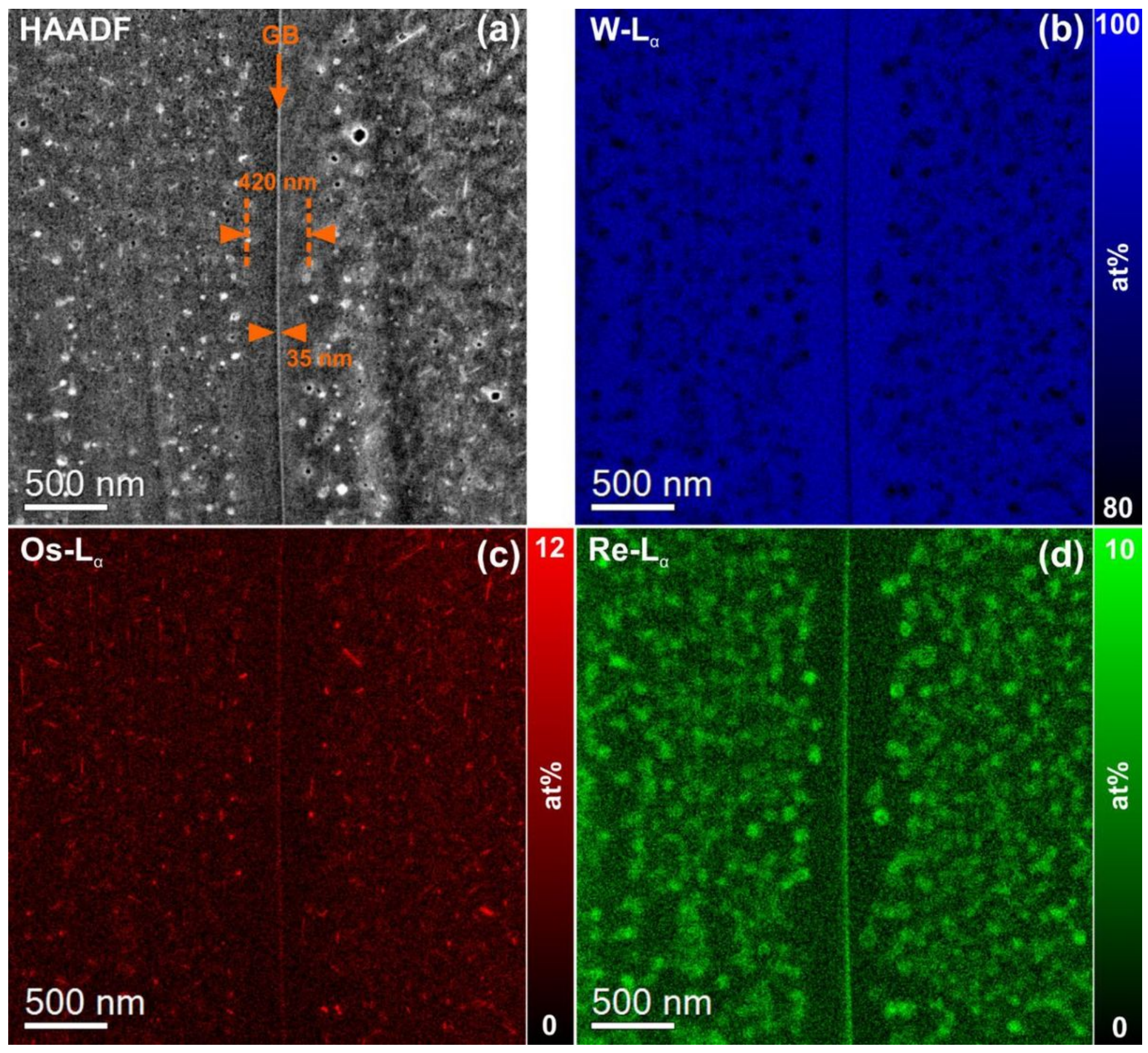

Figure 6

STEM-EDX mapping of a grain boundary. (a) STEM-HAADF image. The position of the $\mathrm{W}$ grain boundary (GB) is indicated in the HAADF image. Elemental maps of (b) W, (c) Os, and (d) Re display the location of elements with respect to the sample morphology. 


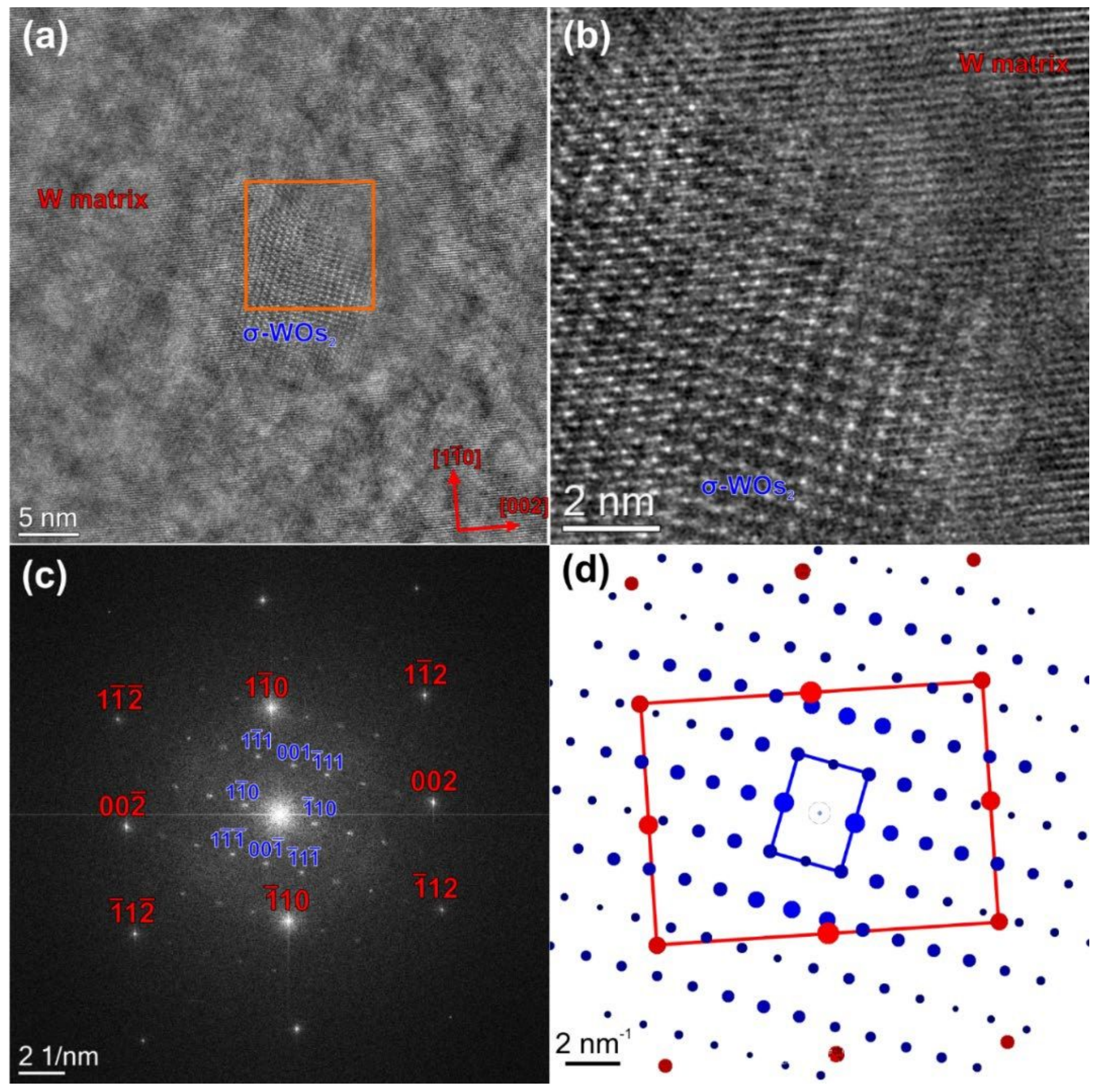

Figure 7

(a) High-resolution phase contrast image of a spherical-shaped Os-rich precipitate, which is identified as the WOs2 $\sigma$-phase. Magnified view (b) and diffractogram (FFT) (c) of the region delimited by the orange square in (a). (d) Simulated model of the diffractogram. 


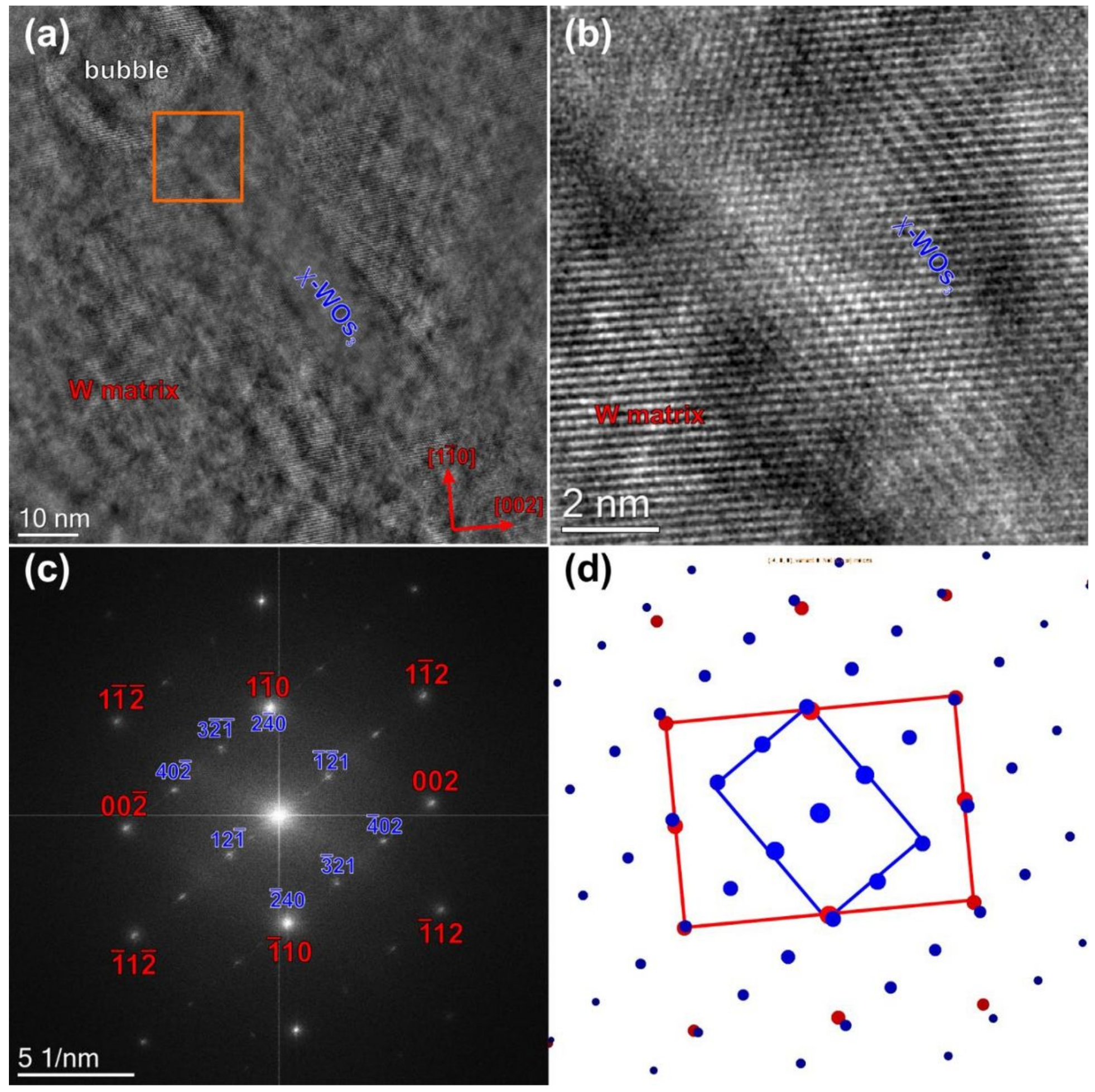

Figure 8

(a) High-resolution phase contrast image of a rod-shaped Os-rich precipitate, which is identified as the WOs3 $x$-Phase. Magnified view (b) and diffractogram (FFT) (c) of the region delimited by the orange square in (a). Simulated model of the diffractogram (d). 


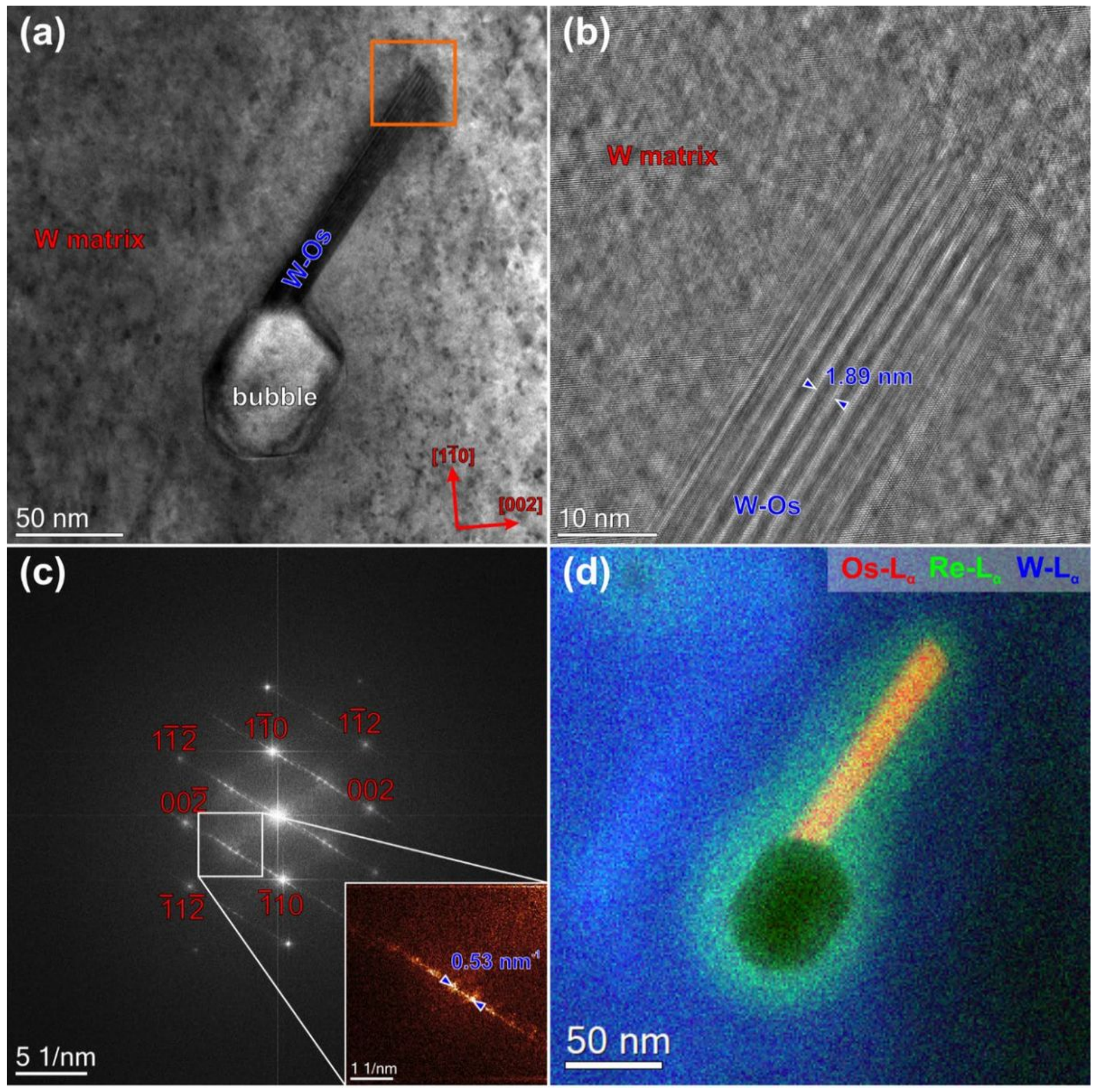

Figure 9

(a) TEM bright-field image of a faceted cavity attached to a W-Os rod. (b) High-resolution phase contrast image of the W-Os phase exhibiting Moiré fringes in the Os-rich part. (c) Diffractogram (FFT) of (b). (d) Composite STEM-EDX elemental map of the same sample region. 

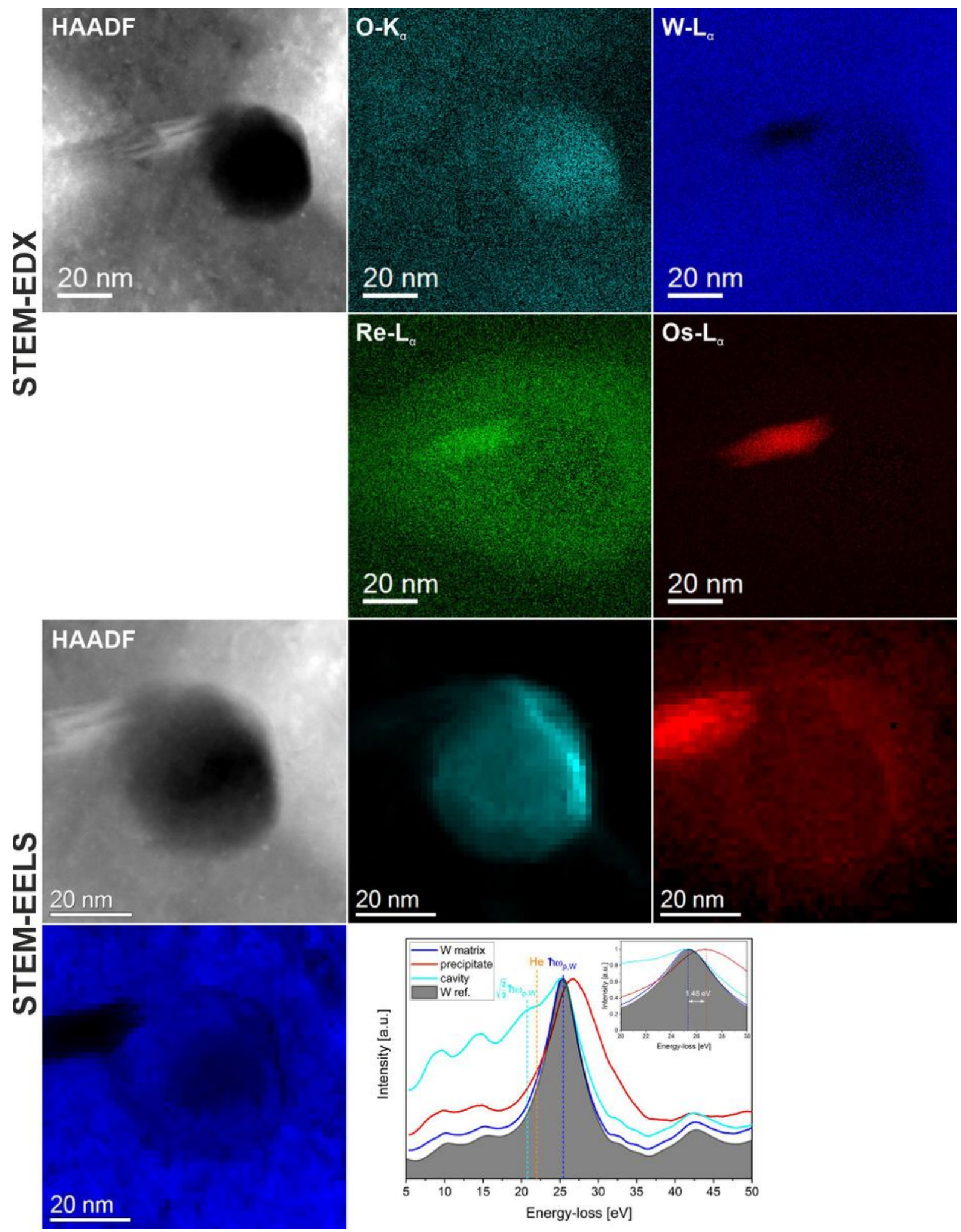

Figure 10

Combined STEM-EDX and low-loss STEM-EELS measurement of a individual cavity. The intensities of the STEM-EELS maps was obtained by NLLS fitting of the peaks located in the W plasmon peak area. 